\title{
Dairy Consumption and Risk of Metabolic Syndrome: Results from Korean Population and Meta-Analysis
}

\author{
Shaoyue Jin $\mathbb{1}$ and Youjin Je * \\ Department of Food and Nutrition, Kyung Hee University, Seoul 02447, Korea; shaoyue666@gmail.com \\ * Correspondence: youjinje@khu.ac.kr; Tel.: +82-2-961-0258
}

Citation: Jin, S.; Je, Y. Dairy

Consumption and Risk of Metabolic Syndrome: Results from Korean Population and Meta-Analysis. Nutrients 2021, 13, 1574. https: / / doi.org/10.3390/nu13051574

Academic Editor: Breige McNulty

Received: 5 April 2021

Accepted: 6 May 2021

Published: 8 May 2021

Publisher's Note: MDPI stays neutral with regard to jurisdictional claims in published maps and institutional affiliations.

Copyright: (c) 2021 by the authors. Licensee MDPI, Basel, Switzerland. This article is an open access article distributed under the terms and conditions of the Creative Commons Attribution (CC BY) license (https:/ / creativecommons.org/licenses/by/ $4.0 /)$.

\begin{abstract}
Dairy consumption has been associated with decreased risk of metabolic syndrome (MetS) in previous studies, but the association may be different according to each type of dairy products and its subgroups. Thus, we conducted an updated meta-analysis of observational studies to examine the association between various dairy products and risk of MetS. The PubMed and Web of Science databases were searched for eligible studies published up to February 2021. In addition, we included unpublished results from Korea National Health and Nutrition Examination Survey, 2013-2018, including 23,319 Korean adults and the elderly. A total of 35 studies (12 cohort studies and 25 cross-sectional studies) with 398,877 subjects were included in the meta-analysis. The pooled relative risks (RR) of MetS for the highest versus lowest categories of dairy consumption was 0.80 [95\% confidence interval (CI): 0.72-0.88]. For the type of dairy products, there were also significant inverse associations with milk (RR: 0.83; 95\% CI: 0.78-0.89) and yogurt consumption (RR: 0.89 ; 95\% CI: 0.83-0.95). For cheese consumption, however, no significant association was found (RR: $0.98 ; 95 \% \mathrm{CI}$ : 0.86-1.11). Our findings suggest that milk and yogurt consumption is inversely associated with the risk of MetS, but not cheese consumption.
\end{abstract}

Keywords: metabolic syndrome; dairy products; milk; yogurt; cheese

\section{Introduction}

Metabolic syndrome (MetS) is a complex of interrelated risk factors for cardiovascular disease and diabetes, including raised blood pressure, raised triglycerides, lowered highdensity lipoprotein cholesterol, raised fasting glucose, and central obesity [1]. About $20-25 \%$ of the world's adult population has MetS [2]. Compared to people without MetS, those with MetS are twice as likely to increase the risk of cardiovascular disease (CVD) and CVD mortality, and 1.5 times as likely to increase the risk for all-cause mortality [3]. The relationship between dairy consumption and the risk of MetS has already been extensively investigated, and there are some hypotheses that some ingredients in dairy products, including a variety of minerals, dairy proteins, and dairy fats, may have beneficial effects on MetS [4,5].

Four meta-analyses on dairy consumption and MetS risk have been previously conducted [6-9]. Two meta-analyses were mainly conducted on total dairy consumption [6,7], while the other two meta-analyses reported some results of individual dairy products but did not conduct subgroup analyses of individual dairy products [8,9]. Previous studies did not perform stratified analyses by sex and criteria for MetS, and previous meta-analyses pointed out the different MetS criteria as a limitation [7-9]. In addition, there were some observational studies on cheese consumption and risk of MetS, but no meta-analysis of cheese consumption on risk of MetS has been conducted to date. Also, we need to conduct an updated meta-analysis of observational studies with the most recent data and dose-response analyses.

Therefore, we systematically reviewed and performed a comprehensive meta- analysis to quantitatively evaluate the association between total dairy products and subtype of 
dairy products, such as milk, yogurt and cheese consumption, and MetS. In addition, we analyzed the associations of each type of dairy products with the risk of MetS using the data of Korea National Health and Nutrition Examination Survey (KNHANES) and included these new results in our meta-analysis.

\section{Materials and Methods}

\subsection{Korea National Health and Nutrition Examination Survey (KNHANES) Data}

2.1.1. Study Participants

The present study was based on the KNHANES data, which was conducted by the Korea Centers for Disease Control and Prevention (KCDC) under the Korean Ministry of Health and Welfare. A total of 47,217 participants were collected from the KNHANES 2013-2018, and among them, 39,642 subjects who completed the health interview, health examination, and nutrition survey were included. The following individuals were subsequently excluded: 8453 participants who were $<19$ years old; 2916 participants who had previously diagnosed with a stroke, myocardial infarction, or cancer; 409 participants who were pregnant or lactating women; 536 participants who had extreme energy intake ( $<500$ or $>5000 \mathrm{kcal} /$ day); 1331 participants who had no fasting status at blood test; 1164 participants who had incomplete information on biomarkers; 1514 participants who had missing data on alcohol consumption, smoking, or moderate or vigorous physical activity. Finally, 23,319 participants (18,206 adults, 5113 elderly people) were included in the analysis of MetS. The Institutional Review Board of the KCDC provided formal ethics approval for the KNHANES dataset (2013-07CON-03-4C, 2013-12EXP-03-5C, 2018-01-03-P-A), and informed consent was obtained for each participant in the survey.

\subsubsection{Dietary Assessment}

Milk and yogurt consumption was assessed from a $24 \mathrm{~h}$ dietary recall based on the coding scheme of KNHANES [10,11]. According to the 2015 Dietary Reference Intake for Koreans (KDRI), one serving was defined as $200 \mathrm{~g}$ for milk, $150 \mathrm{~g}$ for liquid yogurt, $100 \mathrm{~g}$ for semisolid yogurt [12]. The consumption of milk and yogurt was categorized into three groups $(0,<1, \geq 1$ serving/day).

\subsubsection{Assessment of Metabolic Syndrome (MetS)}

The MetS was defined using the National Cholesterol Education Program Adult Treatment Panel III (NCEP ATP III) [13] and the Korean Society for the study of Obesity [14] criteria. The MetS was diagnosed when at least three of the following five components were present: (1) abdominal obesity (waist circumference $\geq 90 \mathrm{~cm}$ for men and $\geq 85 \mathrm{~cm}$ for women); (2) elevated blood pressure (systolic blood pressure $\geq 130 \mathrm{mmHg}$ or diastolic blood pressure $\geq 85 \mathrm{mmHg}$ or use of antihypertensive medication); (3) low high-density lipoprotein (HDL)-cholesterol (fasting HDL-cholesterol $<40 \mathrm{mg} / \mathrm{dL}$ for men and $<50 \mathrm{mg} / \mathrm{dL}$ for women); (4) hypertriglyceridemia (fasting triglyceride $\geq 150 \mathrm{mg} / \mathrm{dL}$ ); (5) hyperglycemia (fasting plasma glucose $\geq 100 \mathrm{mg} / \mathrm{dL}$ or physician's diagnosis or use of insulin or oral antidiabetic medication).

\subsubsection{Confounding Variables}

Information on the demographic and lifestyle factors including age, sex, socioeconomic, smoking status, alcohol consumption, and physical activity were obtained by personal interview or using a self-administered questionnaire. Education level was classified as " $\leq$ middle school", "high school", and " $\geq$ college". Household income level was categorized into the lowest, lower-middle, upper-middle, and highest. Smoking status was divided into the non-smoker, former smoker, and current smoker. We surveyed drinking experience in the past year, and alcohol consumption was categorized into never/rarely, 1-4 times/month, and $\geq 2$ times/week. Physical activity was assessed by the practice of aerobic activity. The practice of aerobic activity was defined as $\geq 150 \mathrm{~min} /$ week of moderate physical activity, $\geq 75 \mathrm{~min} /$ week of vigorous physical activity, or $\geq 150 \mathrm{~min} /$ week of a 
combination of moderate and vigorous physical activity ( 1 min of vigorous physical activity was considered 2 min of moderate physical activity). Furthermore, we also calculated total energy intake and used it as a continuous variable.

\subsubsection{Statistical Analysis}

The KNHANES data were combined from 2013-2018 while considering the cluster sampling design for the survey according to the guidelines provided by KCDC [10,11]. The general characteristics of the participants were described as shown in Supplemental Tables S1-S3. Continuous variables such as age, body mass index (BMI), total energy intake, nutrient intake, and milk and yogurt intake were expressed as means \pm standard errors (SEs), and categorical variables such as sex, education level, household income, smoking status, alcohol consumption and physical activity were expressed as numbers of participants and percentages. The differences in characteristics according to milk and yogurt consumption were evaluated and the PROC SURVEYREG procedure for continuous variables and the chi-square test for categorical variables were used. In addition, the PROC SURVEYLOGISTIC procedure was used for logistic regression analysis and age-adjusted and multivariable-adjusted odds ratios (ORs) and 95\% confidence intervals (CIs) of MetS were calculated according to the milk and yogurt consumption. All statistical analyses were performed using SAS software, version 9.4 (SAS Institute Inc., Cary, NC, USA). A two-tailed $p$ value $<0.05$ was considered to indicate statistical significance.

\subsection{Systematic Review, Meta-Analysis}

\subsubsection{Literature Search and Study Selection}

Eligible studies were searched from published up to February 2021 in PubMed and ISI Web of Science databases. The following search terms were used: "(milk, yogurt, yoghurt, cheese, or dairy) combined with (metabolic syndrome, syndrome $\mathrm{X}$, or insulin resistant syndrome)". In addition, a manual search of the reference lists of all relevant studies was conducted, and the articles were reviewed to find other qualified studies. This meta-analysis included prospective cohort studies and cross-sectional studies to report the association between consumption of dairy products and risk of MetS, and included articles published in full-length and in English. To be included in the analysis, the study reported relative risk (RR) and 95\% confidence intervals (CI). If there were several publications from the same study, we selected the publication with the largest number of cases or selected a cohort study instead of a cross-sectional study.

\subsubsection{Data Extraction}

Two investigators (S.J. and Y.J.) extracted data independently, according to the metaanalysis of observational studies in epidemiology (MOOSE) guidelines [15], and any disagreements were resolved by further discussion and referencing the original articles. The following data were extracted from each study: first author's last name, year of publication, geographic region, study design, follow-up period or study period, baseline age, sex, sample size, number of cases, type of dairy products, adjustment factors, and RRs and $95 \%$ CIs for the association between consumption of dairy products and MetS across various categories of exposure or per unit change in exposure. If the study provided more than one RR from age-adjusted models to different multivariate models, we used the RR of the multivariate model to make the most full adjustment for confounders.

\subsubsection{Statistical Analysis}

The pooled RRs and $95 \%$ CIs of the highest versus lowest dairy products consumptions were calculated from the original studies. The natural logarithm of the RRs from the original study were combined through using the DerSimonian and Laird random-effects models, which incorporate both within- and between-study variations [16]. If the original study did not report the lowest level as a reference, we recalculated the RR and its 95\% CI [17-21]. When a study reports the results of normal weight at base line and overweight or obese at 
baseline, we first combined the two results using a fixed-effect model to obtain an overall estimate before combining with other studies [22,23]. If a study reported individual RR for whole milk and low-fat milk but not total milk, we included the effect estimates of whole milk in the main analysis of milk [24,25]. We presented the summary estimates as forest plots. Statistical heterogeneity among the studies was assessed using the $Q$ statistic [26] and inconsistency was quantified through the $I^{2}$ statistic [27]. We conducted subgroup analyses stratified by study design (cohort/cross-sectional), sex, geographic region (Europe/United States/Asia/Oceania), and criteria for MetS (NCEP ATP III/Joint Interim Statement (JIS)/International Diabetes Federation (IDF)/others).

For the dose-response analysis, a generalized least-squares trend (GLST) estimation, based on the method developed by Greenland and Longnecker, was used to compute study-specific slopes from the natural logs of the RRs across intake categories of dairy product consumption [28-30]. The method requires the number of cases and subjects or person-years and RRs with variance estimates for at least three levels of dairy products consumption. For this analysis, prospective cohort studies were included only. The servings, portions, or ounces of dairy product intake per day, week, or month were converted to grams per day for these analyses. According to the standard of 'United States Department of Agriculture Food and Nutrient Database for Dietary Studies' [31], we defined one serving as $177 \mathrm{~g}$ for dairy products, $244 \mathrm{~g}$ for milk, $244 \mathrm{~g}$ for yogurt, and $43 \mathrm{~g}$ for cheese. Finally, potential publication bias was assessed with Begg's [32] and Egger's tests [33]. A two-sided $p$ value $<0.05$ was considered statistically significant. Stata/SE version 14.2 Software (StataCorp, College Station, TX, USA) was used for all statistical analyses.

\section{Results}

\subsection{KNHANES Analysis}

The general characteristics of the study population according to milk and yogurt consumption are shown in Supplementary Tables S1-S3. More than three-quarters of the adult and elderly population did not consume milk or yogurt. The people who consumed higher levels of milk or yogurt had higher calcium intake than non-consumers. For adult and elderly people, frequent milk and yogurt consumers had a higher proportion of total energy intake from fat but had a lower proportion of total energy intake from carbohydrates, while these correlations were stronger in elderly people.

The associations of milk consumption with risk of MetS in adults and elderly people are presented in Table 1. After adjusting for covariates, high milk consumption was associated with $26 \%$ lower odds of MetS in elderly people compared to no milk consumption (OR: $0.74 ; 95 \%$ CI: $0.57-0.96$; $p$ for trend $=0.029$ ), but no association was found in adults (OR: 0.91; 95\% CI: 0.78-1.06; $p$ for trend = 0.246). For the elderly, when stratified by sex, a similar inverse association was found in women (OR: $0.72 ; 95 \%$ CI: $0.52-0.99 ; p$ for trend $=0.058)$, but not in men (OR: 0.83; 95\% CI: 0.56-1.23; $p$ for trend $=0.359)$. For yogurt consumption, however, there was no significant association with risk of MetS (Table 2).

The associations of milk and yogurt consumption with MetS components are shown in Supplementary Tables S4 and S5. For milk consumption, high milk consumption was inversely associated with hypertriglyceridemia in adult men (OR: 0.77; 95\% CI: 0.66-0.90; $p$ for trend $=0.002$ ) and low HDL-cholesterol in elderly women (OR: 0.63; 95\% CI: 0.48-0.84; $p$ for trend $=0.002$ ). For yogurt consumption, high yogurt consumption was inversely associated with hyperglycemia in adult women (OR: 0.76; 95\% CI: $0.61-0.94 ; p$ for trend $=0.006$ ) and elderly men (OR: 0.65; 95\% CI: 0.43-0.98; $p$ for trend $=0.049$ ). 
Table 1. Multivariable-adjusted odds ratio (ORs) and 95\% confidence intervals (CIs) for metabolic syndrome according to milk consumption in Korean adult and elderly population.

\begin{tabular}{|c|c|c|c|c|}
\hline & \multicolumn{3}{|c|}{ Milk Consumption (Servings/Day) } & \multirow{2}{*}{$p$ Trend $^{1}$} \\
\hline & 0 & $0<$ to $<1$ & $\geq 1$ & \\
\hline \multicolumn{5}{|l|}{ All adults (4005 cases $/ 18,206$ subjects) } \\
\hline No. of cases/subjects & $3175 / 13,664$ & $313 / 1701$ & $517 / 2841$ & \\
\hline Age-adjusted OR $(95 \% \mathrm{CI})$ & 1.0 (reference) & $0.76(0.65-0.88)$ & $0.78(0.70-0.89)$ & $<0.001$ \\
\hline Multivariable-adjusted OR $(95 \% \mathrm{CI})^{2}$ & 1.0 (reference) & $1.01(0.85-1.20)$ & $0.91(0.78-1.06)$ & 0.246 \\
\hline \multicolumn{5}{|l|}{ Men (2208 cases / 7488 subjects) } \\
\hline No. of cases/subjects & $1775 / 5855$ & $149 / 519$ & $284 / 1114$ & \\
\hline Age-adjusted OR (95\%CI) & 1.0 (reference) & $0.92(0.74-1.15)$ & $0.81(0.69-0.96)$ & 0.013 \\
\hline Multivariable-adjusted OR $(95 \% \mathrm{CI})^{2}$ & 1.0 (reference) & $1.03(0.81-1.32)$ & $0.87(0.71-1.06)$ & 0.204 \\
\hline \multicolumn{5}{|l|}{ Women (1797 cases / 10,718 subjects) } \\
\hline No. of cases/subjects & $1400 / 7809$ & $164 / 1182$ & $233 / 1727$ & \\
\hline Age-adjusted OR $(95 \% \mathrm{CI})$ & 1.0 (reference) & $0.76(0.62-0.93)$ & $0.80(0.67-0.96)$ & 0.002 \\
\hline Multivariable-adjusted OR $(95 \% \mathrm{CI})^{2}$ & 1.0 (reference) & $0.96(0.76-1.20)$ & $0.95(0.77-1.18)$ & 0.608 \\
\hline \multicolumn{5}{|c|}{ All elderly people (2320 cases / 5113 subjects) } \\
\hline No. of cases/subjects & $1941 / 4196$ & $175 / 391$ & $204 / 526$ & \\
\hline Age-adjusted OR (95\%CI) & 1.0 (reference) & $0.97(0.76-1.23)$ & $0.73(0.59-0.92)$ & 0.013 \\
\hline Multivariable-adjusted OR $(95 \% \mathrm{CI})^{2}$ & 1.0 (reference) & $0.95(0.73-1.24)$ & $0.74(0.57-0.96)$ & 0.029 \\
\hline \multicolumn{5}{|l|}{ Men (859 cases / 2200 subjects) } \\
\hline No. of cases/subjects & $737 / 1862$ & $56 / 136$ & $66 / 202$ & \\
\hline Age-adjusted OR $(95 \% C I)$ & 1.0 (reference) & $1.04(0.69-1.55)$ & $0.73(0.51-1.05)$ & 0.129 \\
\hline Multivariable-adjusted OR $(95 \% \mathrm{CI})^{2}$ & 1.0 (reference) & $0.97(0.61-1.55)$ & $0.83(0.56-1.23)$ & 0.359 \\
\hline \multicolumn{5}{|l|}{ Women (1461 cases / 2913 subjects) } \\
\hline No. of cases/subjects & $1204 / 2334$ & $119 / 255$ & $138 / 324$ & \\
\hline Age-adjusted OR $(95 \% \mathrm{CI})$ & 1.0 (reference) & $0.86(0.63-1.16)$ & $0.69(0.52-0.92)$ & 0.010 \\
\hline Multivariable-adjusted OR $(95 \% \mathrm{CI})^{2}$ & 1.0 (reference) & $0.93(0.67-1.28)$ & $0.72(0.52-0.99)$ & 0.058 \\
\hline \multirow{2}{*}{\multicolumn{5}{|c|}{$\begin{array}{l}1 p \text { value were obtained from a regression model using the PROC SURVEYLOGISTIC procedure. }{ }^{2} \text { Adjusted for age (continuous), sex, body } \\
\text { mass index (BMI, continuous), education ( } \leq \text { middle school, high school, or } \geq \text { college), household income (lowest, lower middle, upper } \\
\text { middle, or highest), smoking (non-smoker, former smoker, or current smoker), alcohol intake (never/rarely, } 1-4 / \text { month, or } \geq 2 / \text { week), } \\
\text { physical activity (yes or no), and total energy (continuous). }\end{array}$}} \\
\hline & & & & \\
\hline & \multicolumn{3}{|c|}{ Yogurt Consumption (Servings/Day) } & \\
\hline & 0 & $0<$ to $<1$ & $\geq 1$ & $p$ Trend $^{-}$ \\
\hline \multicolumn{5}{|l|}{ All adults (4005 cases $/ 18,206$ subjects) } \\
\hline No. of cases/subjects & $3554 / 15,797$ & $220 / 1161$ & $231 / 1248$ & \\
\hline Age-adjusted OR $(95 \% \mathrm{CI})$ & 1.0 (reference) & $0.74(0.61-0.89)$ & $0.74(0.62-0.88)$ & $<0.001$ \\
\hline Multivariable-adjusted OR $(95 \% \mathrm{CI})^{2}$ & 1.0 (reference) & $0.94(0.75-1.17)$ & $0.84(0.70-1.02)$ & 0.065 \\
\hline \multicolumn{5}{|l|}{ Men (2208 cases / 7488 subjects) } \\
\hline No. of cases/subjects & $1999 / 6665$ & $90 / 353$ & $119 / 470$ & \\
\hline Age-adjusted OR (95\%CI) & 1.0 (reference) & $0.83(0.62-1.11)$ & $0.75(0.58-0.96)$ & 0.013 \\
\hline Multivariable-adjusted OR $(95 \% \mathrm{CI})^{2}$ & 1.0 (reference) & $0.97(0.69-1.35)$ & $0.83(0.63-1.08)$ & 0.158 \\
\hline \multicolumn{5}{|l|}{ Women (1797 cases /10,718 subjects) } \\
\hline No. of cases/subjects & $1555 / 9132$ & $130 / 808$ & $112 / 778$ & \\
\hline Age-adjusted OR $(95 \% \mathrm{CI})$ & 1.0 (reference) & $0.78(0.60-1.00)$ & $0.76(0.60-0.96)$ & 0.008 \\
\hline Multivariable-adjusted OR $(95 \% \mathrm{CI})^{2}$ & 1.0 (reference) & $0.88(0.66-1.17)$ & $0.87(0.67-1.12)$ & 0.200 \\
\hline \multicolumn{5}{|c|}{ All elderly people (2320 cases / 5113 subjects) } \\
\hline No. of cases/subjects & $2000 / 4371$ & $187 / 435$ & $133 / 307$ & \\
\hline Age-adjusted OR $(95 \% \mathrm{CI})$ & 1.0 (reference) & $0.85(0.67-1.08)$ & $0.86(0.65-1.12)$ & 0.155 \\
\hline Multivariable-adjusted OR $(95 \% \mathrm{CI})^{2}$ & 1.0 (reference) & $0.80(0.60-1.07)$ & $0.91(0.68-1.21)$ & 0.296 \\
\hline
\end{tabular}


Table 2. cont.

\begin{tabular}{|c|c|c|c|c|}
\hline & \multicolumn{3}{|c|}{ Yogurt Consumption (Servings/Day) } & \multirow{2}{*}{$p$ Trend $^{1}$} \\
\hline & 0 & $0<$ to $<1$ & $\geq \mathbf{1}$ & \\
\hline \multicolumn{5}{|l|}{ Men (859 cases / 2200 subjects) } \\
\hline No. of cases/subjects & $751 / 1918$ & $63 / 168$ & $45 / 114$ & \\
\hline Age-adjusted OR (95\%CI) & 1.0 (reference) & $0.82(0.56-1.19)$ & $0.92(0.59-1.41)$ & 0.512 \\
\hline Multivariable-adjusted OR $(95 \% \mathrm{CI})^{2}$ & 1.0 (reference) & $0.78(0.51-1.21)$ & $0.92(0.59-1.44)$ & 0.507 \\
\hline \multicolumn{5}{|l|}{ Women (1461 cases / 2913 subjects) } \\
\hline No. of cases/subjects & $1249 / 2453$ & $124 / 267$ & $88 / 193$ & \\
\hline Age-adjusted OR (95\%CI) & 1.0 (reference) & $0.84(0.63-1.14)$ & $0.77(0.55-1.09)$ & 0.091 \\
\hline Multivariable-adjusted OR $(95 \% \mathrm{CI})^{2}$ & 1.0 (reference) & $0.81(0.57-1.15)$ & $0.89(0.62-1.28)$ & 0.353 \\
\hline
\end{tabular}

${ }^{1} p$ value were obtained from a regression model using the PROC SURVEYLOGISTIC procedure. ${ }^{2}$ Adjusted for age (continuous), sex, BMI (continuous), education ( $\leq$ middle school, high school, or $\geq$ college), household income (lowest, lower middle, upper middle, or highest), smoking (non-smoker, former smoker, or current smoker), alcohol intake (never/rarely, $1-4 /$ month, or $\geq 2 /$ week), physical activity (yes or no), and total energy (continuous).

\subsection{Systematic Review and Meta-Analysis \\ 3.2.1. Study Characteristics}

A total of 35 studies including 12 cohort studies [22-24,34-44] with 40,943 subjects and 9436 cases and 25 cross-sectional [17-21,25,45-65] studies with 362,196 subjects were included in meta-analyses of the highest versus the lowest dairy product consumption and risk of MetS (Figure 1). Table 3 presents the characteristics of studies included in the meta-analysis. By geographic region, 16 studies were performed in Asia, 9 studies in America, 8 studies in Europe, 1 study in Oceania, and 1 study was a multinational study. The participants were all aged $\geq 18$ years at baseline, and durations of follow-up of cohort studies ranged from 2.05 to 20 years, and the mean follow-up period was 7.22 years. The defined MetS in the studies was mostly from NCEP ATP III, JIS, or IDF criteria. Twenty-one studies used NCEP ATP III or modified NCEP ATP III criteria, 12 studies used JIS criteria, and 4 studies used IDF criteria. The two studies reported the results using several different MetS criteria [38,54]. All of studies adjusted for age, and most of the studies adjusted for BMI $\left(\mathrm{kg} / \mathrm{m}^{2}\right)$, smoking, alcohol intake, physical activity, socioeconomic status, and total energy intake.
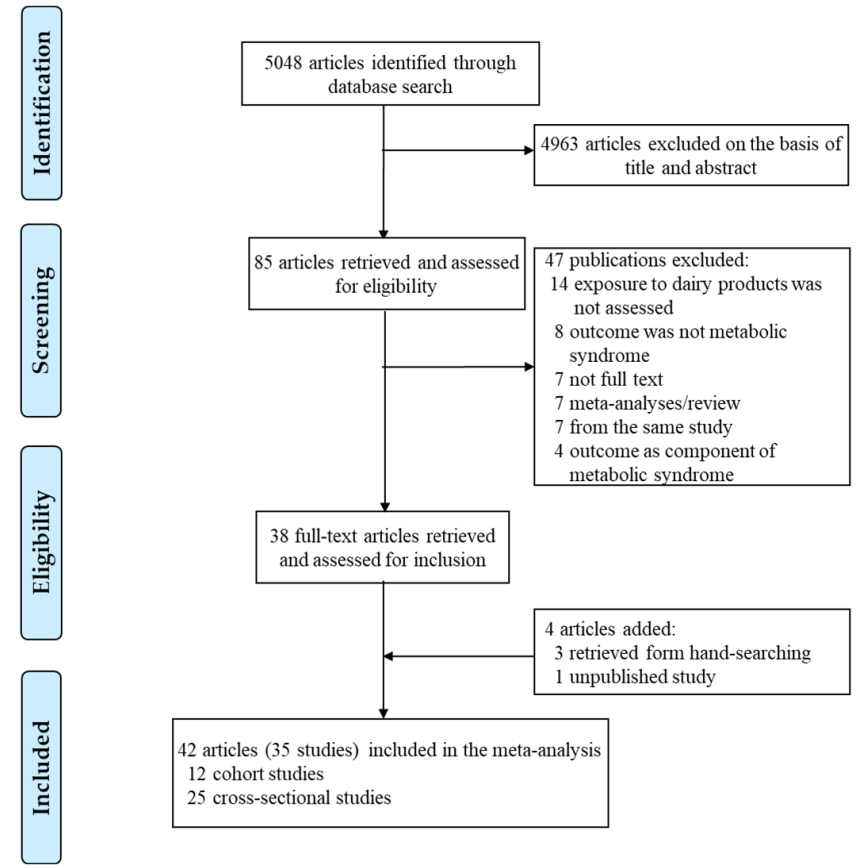

Figure 1. Flow chart of the study selection. 
Table 3. Characteristics of prospective cohort/cross-sectional studies included in the meta-analysis of dairy product intake and metabolic syndrome.

\begin{tabular}{|c|c|c|c|c|c|c|c|}
\hline $\begin{array}{l}\text { First Author } \\
\text { (Year) }\end{array}$ & $\begin{array}{c}\text { Country (Study } \\
\text { Name) }\end{array}$ & $\begin{array}{l}\text { Study } \\
\text { Design }\end{array}$ & $\begin{array}{c}\text { Age } \\
\text { (Years) }\end{array}$ & Subjects & $\begin{array}{l}\text { Criteria for } \\
\text { Metabolic } \\
\text { Syndrome }\end{array}$ & Exposure Category & Adjustment Factors \\
\hline $\begin{array}{l}\text { Pereira } \\
(2002)[22]\end{array}$ & $\begin{array}{l}\text { USA (Coronary } \\
\text { Artery Risk } \\
\text { Development in } \\
\text { Young Adults } \\
\text { study, CARDIA) }\end{array}$ & Cohort & $18-30$ & $467 / 3157$ & $\begin{array}{l}\geq 2 \text { of the } 4 \\
\text { components: } \\
\text { abnormal } \\
\text { glucose } \\
\text { homeostasis, } \\
\text { obesity, elevated } \\
\text { BP, and } \\
\text { dyslipidemia. }\end{array}$ & $\begin{array}{l}\text { Dairy products } \\
0-<10 \text { (ref.), } 10-<16, \\
16-<24,24-<35, \geq 35 \\
\text { times/week } \\
\text { Milk } \\
1 \text { daily increment } \\
\text { Yogurt } \\
1 \text { daily increment }\end{array}$ & $\begin{array}{l}\text { Age, sex, BMI, race, calorie } \\
\text { intake/day, study center, } \\
\text { education, smoking, alcohol, } \\
\text { PA, vitamin supplement, } \\
\text { polyunsaturated fat, caffeine, } \\
\text { fiber/1000 calories, whole and } \\
\text { refined grains, meat, fruit, } \\
\text { vegetables, soda, magnesium, } \\
\text { Ca and vitamin D }\end{array}$ \\
\hline $\begin{array}{l}\text { Damiăo } \\
\text { (2006) [34] }\end{array}$ & Brazil & Cohort & $40-79$ & $57 / 151$ & NCEP ATP III & $\begin{array}{l}\text { Milk } \\
12.4 \text { (ref.), 141.7, } 223.7 \\
\text { g/day }\end{array}$ & $\begin{array}{l}\text { Age, sex, smoking, PA, } \\
\text { education, alcohol, total energy } \\
\text { intake }\end{array}$ \\
\hline $\begin{array}{l}\text { Lutsey } \\
(2008)[35]\end{array}$ & $\begin{array}{l}\text { USA } \\
\text { (Atherosclerosis } \\
\text { Risk in } \\
\text { Communities } \\
\text { study, ARIC) }\end{array}$ & Cohort & $45-64$ & $3782 / 9514$ & $\begin{array}{l}\text { American Heart } \\
\text { Association } \\
\text { guidelines }\end{array}$ & $\begin{array}{l}\text { Dairy products } \\
0.28 \text { (ref.), } 0.93,1.29, \\
1.94,3.30 \\
\text { servings/day }\end{array}$ & $\begin{array}{l}\text { Age, sex, race, education, } \\
\text { smoking, center, total calories, } \\
\text { PA, pack-years, meat, dairy, } \\
\text { vegetables, fruits, and whole } \\
\text { and refined grains }\end{array}$ \\
\hline $\begin{array}{l}\text { Snijder } \\
(2008)[36]\end{array}$ & $\begin{array}{l}\text { Netherlands } \\
\text { (Hoorn study) }\end{array}$ & Cohort & $50-75$ & $215 / 1124$ & NCEP ATP III & $\begin{array}{l}\text { Dairy products } \\
\text { Quartile (Q) 1(ref.), } \\
\text { Q4 }\end{array}$ & $\begin{array}{l}\text { Age, sex, smoking, alcohol, } \\
\text { total energy, PA }\end{array}$ \\
\hline $\begin{array}{l}\text { Duffey } \\
(2010) \text { [37] }\end{array}$ & $\begin{array}{l}\text { USA (Coronary } \\
\text { Artery Risk } \\
\text { Development in } \\
\text { Young Adults } \\
\text { study, CARDIA) }\end{array}$ & Cohort & $18-30$ & $459 / 3596$ & NCEP ATP III & $\begin{array}{l}\text { Whole fat milk } \\
\text { Moving from } 1 \\
\text { quartile to the next }\end{array}$ & $\begin{array}{l}\text { Age, race, sex, CARDIA exam } \\
\text { center, weight, smoking, total } \\
\text { PA, energy from food, the } 3 \\
\text { other beverages, and alcohol }\end{array}$ \\
\hline $\begin{array}{l}\text { Fumeron } \\
\text { (2011) [38] }\end{array}$ & $\begin{array}{l}\text { France } \\
\text { (Epidemiological } \\
\text { Study on the } \\
\text { Insulin } \\
\text { Resistance } \\
\text { Syndrome, } \\
\text { DESIR) }\end{array}$ & Cohort & $30-65$ & $452 / 3435$ & $\begin{array}{l}\text { NCEP ATP III } \\
\text { IDF }\end{array}$ & $\begin{array}{l}\text { Dairy products } \\
\text { Per a change from } 1 \\
\text { category to the next. } \\
\text { Cheese } \\
\text { Per a change from } 1 \\
\text { category to the next. }\end{array}$ & $\begin{array}{l}\text { Age, sex, smoking, total fat } \\
\text { intake, PA, BMI }\end{array}$ \\
\hline Lin (2013) [39] & Taiwan & Cohort & $\geq 65$ & $206 / 888$ & NCEP ATP III & $\begin{array}{l}\text { Milk } \\
\text { No (ref.), yes }\end{array}$ & $\begin{array}{l}\text { Age, sex, smoking, alcohol, } \\
\text { serum creatinine, uric acid, } \\
\text { ALT, urine protein, initial MetS } \\
\text { score, exercise, teeth brushing, } \\
\text { vegetable }\end{array}$ \\
\hline Louie (2013) [40] & $\begin{array}{l}\text { Australia (Blue } \\
\text { Mountains Eye } \\
\text { Study, BMES) }\end{array}$ & Cohort & $\geq 49$ & $155 / 1807$ & IDF & $\begin{array}{l}\text { Dairy products } \\
0.5 \text { (ref.), } 1.2,1.8,3.1 \\
\text { servings/day }\end{array}$ & $\begin{array}{l}\text { Age, sex, smoking, PA, dietary } \\
\text { glycemic load, fibre from } \\
\text { vegetables, family history, total } \\
\text { energy, Ca }\end{array}$ \\
\hline Babio (2015) [41] & $\begin{array}{l}\text { Spain (Prevenci } \\
\text { 'on con Dieta } \\
\text { Mediterr'anea, } \\
\text { PREDIMED) }\end{array}$ & Cohort & $55-80$ & $930 / 1868$ & JIS & $\begin{array}{l}\text { Dairy products } \\
207 \text { (ref.), 354, } 577 \\
\text { g/day } \\
\text { Milk } \\
120 \text { (ref.), 222, } 462 \\
\text { g/day } \\
\text { Yogurt } \\
7 \text { (ref.), } 70,127 \mathrm{~g} / \text { day } \\
\text { Cheese } \\
11 \text { (ref.), 28, } 51 \mathrm{~g} \text { / day }\end{array}$ & $\begin{array}{l}\text { Age, sex, intervention group, } \\
\text { BMI, leisure time PA, smoking, } \\
\text { use of hypoglycemic, } \\
\text { antihypertensive, } \\
\text { hypolipidemic, insulin } \\
\text { treatment at baseline, } \\
\text { vegetables, fruit, legumes, } \\
\text { cereals, red meat, fish, nuts, } \\
\text { cookies, olive oil, alcohol, } \\
\text { prevalence of metabolic } \\
\text { syndrome components at } \\
\text { baseline. }\end{array}$ \\
\hline $\begin{array}{l}\text { Sayón-Orea } \\
\text { (2015) [42] }\end{array}$ & $\begin{array}{l}\text { Spain } \\
\text { (Seguimiento } \\
\text { Universidad de } \\
\text { Navarra, SUN) }\end{array}$ & Cohort & $20-90$ & $306 / 8063$ & JIS & $\begin{array}{l}\text { Yogurt } \\
0-250 \text { (ref.), } \\
>250-<875, \geq 875 \\
\text { g/week }\end{array}$ & $\begin{array}{l}\text { Age, sex, smoking, alcohol, } \\
\text { baseline weight, total energy, } \\
\text { red meat, soft drinks, fast food, } \\
\text { french fries, mediterranean } \\
\text { diet, PA, sedentary behavior, } \\
\text { hours sitting, snacking } \\
\text { between meals, following } \\
\text { special diet }\end{array}$ \\
\hline Kim (2017) [43] & $\begin{array}{l}\text { Korea (Korean } \\
\text { Genome and } \\
\text { Epidemiology } \\
\text { Study, KoGES) }\end{array}$ & Cohort & $40-69$ & $2103 / 5510$ & NCEP ATP III & $\begin{array}{l}\text { Dairy products } \\
\text { None (ref.), }<1,1-<4, \\
4-\leq 7,>7 \\
\text { servings/week } \\
\text { Milk } \\
\text { None (ref.), }<1,1-<4, \\
4-\leq 7,>7 \\
\text { servings/week } \\
\text { Yogurt } \\
\text { None (ref.), }<1,1 \leq \text { to } \\
<4, \geq 4 \text { servings/week }\end{array}$ & $\begin{array}{l}\text { Age, sex, BMI, smoking, } \\
\text { alcohol, residential location, } \\
\text { educational, household } \\
\text { income, PA, energy, } \\
\text { energy-adjusted Ca and fibre }\end{array}$ \\
\hline
\end{tabular}


Table 3. cont.

\begin{tabular}{|c|c|c|c|c|c|c|c|}
\hline $\begin{array}{l}\text { First Author } \\
\text { (Year) }\end{array}$ & $\begin{array}{l}\text { Country (Study } \\
\text { Name) }\end{array}$ & $\begin{array}{c}\text { Study } \\
\text { Design }\end{array}$ & $\begin{array}{c}\text { Age } \\
\text { (Years) }\end{array}$ & Subjects & $\begin{array}{l}\text { Criteria for } \\
\text { Metabolic } \\
\text { Syndrome }\end{array}$ & Exposure Category & Adjustment Factors \\
\hline $\begin{array}{l}\text { Beydoun } \\
\text { (2018) [44] }\end{array}$ & $\begin{array}{l}\text { USA (Healthy } \\
\text { Aging in } \\
\text { Neighborhoods } \\
\text { of Diversity } \\
\text { across the Life } \\
\text { Span, HANDLS) }\end{array}$ & Cohort & $30-64$ & $173 / 1371$ & NCEP ATP III & $\begin{array}{l}\text { Milk } \\
\text { Per cup equivalent } \\
\text { Yogurt } \\
\text { Per fl oz equivalent } \\
\text { Cheese } \\
\text { Per oz equivalent }\end{array}$ & $\begin{array}{l}\text { Age, sex, race, smoking, } \\
\text { alcohol, socio-economic status, } \\
\text { energy intake at baseline, } \\
\text { current drug use and self-rated } \\
\text { health, energy intake, total } \\
\text { fruit, deep yellow vegetables, } \\
\text { dark green vegetables, } \\
\text { non-whole grains, legumes, } \\
\text { whole grains, nuts/seeds, soya, } \\
\text { total meat/poultry/fish, eggs, } \\
\text { discretionary solid fat, } \\
\text { discretionary oils, added } \\
\text { sugars and mg of caffeine. }\end{array}$ \\
\hline $\begin{array}{l}\text { Cheraghi } \\
(2018)[24]\end{array}$ & $\begin{array}{l}\text { Iran (Tehran } \\
\text { Lipid and } \\
\text { Glucose Study, } \\
\text { TLGS) }\end{array}$ & Cohort & $\geq 20$ & $590 / 3616$ & JIS & $\begin{array}{l}\text { Whole fat milk } \\
\text { None (ref.), } 1 \\
\text { cup/day } \\
\text { Yogurt } \\
\text { None (ref.), } 1 / 2 \\
\text { cup/day } \\
\text { Cheese } \\
\text { None (ref.), } 1 \mathrm{oz} / \text { day }\end{array}$ & $\begin{array}{l}\text { Age, sex, cancer history, } \\
\text { hospitalisation } \\
\text { status, education, BMI, marital } \\
\text { status, smoking, calories, } 95 \\
\text { foods and } 12 \text { nutrients }\end{array}$ \\
\hline $\begin{array}{l}\text { Mirmiran } \\
(2020)[23]\end{array}$ & $\begin{array}{l}\text { Iran (Tehran } \\
\text { Lipid and } \\
\text { Glucose Study, } \\
\text { TLGS) }\end{array}$ & Cohort & $\geq 19$ & $368 / 1114$ & JIS & $\begin{array}{l}\text { Dairy products } \\
\text { Per } 1 \text { standard } \\
\text { deviation }\end{array}$ & $\begin{array}{l}\text { Age, sex, academic educations, } \\
\text { baseline BMI, BMI-change, and } \\
\text { energy intakes. }\end{array}$ \\
\hline
\end{tabular}

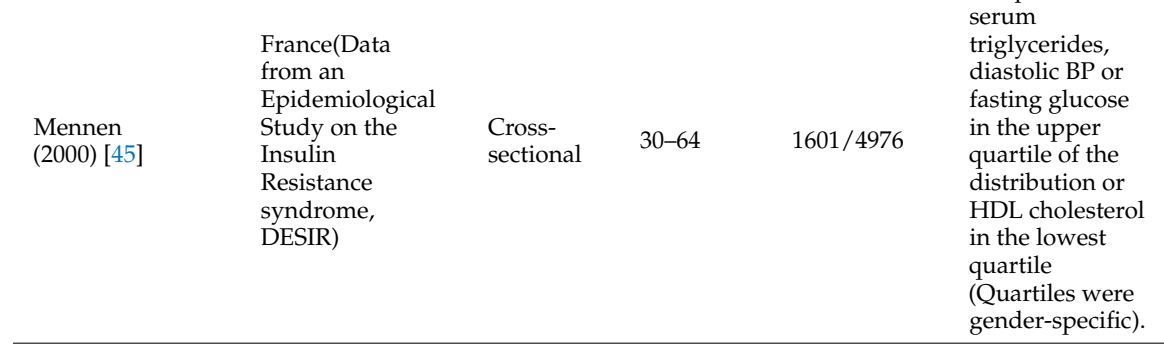

\begin{tabular}{|c|c|c|c|c|c|c|c|}
\hline $\begin{array}{l}\text { Azadbakht } \\
\text { (2005) [46] }\end{array}$ & $\begin{array}{l}\text { Iran (Tehran } \\
\text { Lipid and } \\
\text { Glucose Study, } \\
\text { TLGS) }\end{array}$ & $\begin{array}{l}\text { Cross- } \\
\text { sectional }\end{array}$ & $18-74$ & 827 & NCEP ATP III & $\begin{array}{l}\text { Dairy products } \\
<1.7 \text { (ref.), } 1.7-2.3, \\
2.3-3.1, \geq 3.1 \\
\text { servings/day }\end{array}$ & $\begin{array}{l}\text { Age, BMI, total energy, percent } \\
\text { of energy from fat, smoking, } \\
\text { use of BP and estrogen } \\
\text { medication, PA, food group, } \\
\mathrm{Ca} \text {, and protein intake }\end{array}$ \\
\hline $\begin{array}{l}\text { Lawlor } \\
\text { (2005) [17] }\end{array}$ & $\begin{array}{l}\text { UK (British } \\
\text { Women's Health } \\
\text { Study) }\end{array}$ & $\begin{array}{l}\text { Cross- } \\
\text { sectional }\end{array}$ & $60-79$ & 4024 & WHO & $\begin{array}{l}\text { Milk } \\
\text { Non milk drinker(ref), } \\
\text { milk drinker }\end{array}$ & Age \\
\hline Liu (2005) [47] & $\begin{array}{l}\text { USA (Women's } \\
\text { Health Study) }\end{array}$ & $\begin{array}{l}\text { Cross- } \\
\text { sectional }\end{array}$ & $\geq 45$ & 10,066 & NCEP ATP III & $\begin{array}{l}\text { Dairy products } \\
<0.91 \text { (ref.), } 0.91-1.41, \\
1.42-1.99,2.00-3.00, \\
>3.00 \text { servings/day } \\
\text { Milk } \\
<0.13 \text { (ref.), } 0.13-0.43, \\
0.44-0.93,0.94-1.07, \\
>1.08 \text { servings/day }\end{array}$ & $\begin{array}{l}\text { Age, smoking, alcohol, total } \\
\text { calorie intake, and randomized } \\
\text { treatment assignment, exercise, } \\
\text { total calories, multivitamin, } \\
\text { family history, dietary intakes } \\
\text { of total fat, cholesterol, protein, } \\
\text { and glycemic load }\end{array}$ \\
\hline $\begin{array}{l}\text { Elwood } \\
\text { (2007) [48] }\end{array}$ & $\begin{array}{l}\text { UK (Caerphilly } \\
\text { Cohort Study) }\end{array}$ & $\begin{array}{l}\text { Cross- } \\
\text { sectional }\end{array}$ & $45-59$ & 2375 & WHO & $\begin{array}{l}\text { Milk } \\
\text { Little or none (ref.), } \\
<1 / 2,1 / 2-1,>1 \text { pint }\end{array}$ & Age, social class and smoking \\
\hline $\begin{array}{l}\text { Ruidavets } \\
\text { (2007) [49] }\end{array}$ & France & $\begin{array}{l}\text { Cross- } \\
\text { sectional }\end{array}$ & $45-64$ & 912 & NCEP ATP III & $\begin{array}{l}\text { Dairy products } \\
\text { Q1 (ref.), Q2, Q3, Q4, } \\
\text { Q5 }\end{array}$ & $\begin{array}{l}\text { Age, centre, smoking, alcohol, } \\
\text { PA, energy intake, education, } \\
\text { drugs for hypertension and } \\
\text { dyslipidaemia, dieting, and } \\
\text { diet quality index }\end{array}$ \\
\hline $\begin{array}{l}\text { Beydoun } \\
\text { (2008) [25] }\end{array}$ & $\begin{array}{l}\text { USA(National } \\
\text { Health and } \\
\text { Nutrition } \\
\text { Examination } \\
\text { Survey, } \\
\text { NHANES) }\end{array}$ & $\begin{array}{l}\text { Cross- } \\
\text { sectional }\end{array}$ & $\geq 18$ & 4519 & NCEP ATP III & $\begin{array}{l}\text { Dairy products } \\
\text { Each daily servings } \\
\text { Whole milk } \\
\text { per } 100 \mathrm{~g} \\
\text { Yogurt } \\
\text { Each daily servings } \\
\text { Cheese } \\
\text { Each daily servings }\end{array}$ & $\begin{array}{l}\text { Age, sex, ethnicity, } \\
\text { socioeconomic status, energy } \\
\text { intake, PA, alcohol, total fruit, } \\
\text { deep yellow vegetables, dark } \\
\text { green vegetables, non-whole } \\
\text { grains, whole grains, legumes, } \\
\text { nuts/seeds, soy, total } \\
\text { meat/poultry/fish, eggs, } \\
\text { discretionary solid fat, } \\
\text { discretionary oils, added } \\
\text { sugars, and mg of caffeine. }\end{array}$ \\
\hline
\end{tabular}


Table 3. cont

\begin{tabular}{|c|c|c|c|c|c|c|c|}
\hline $\begin{array}{l}\text { First Author } \\
\text { (Year) }\end{array}$ & $\begin{array}{l}\text { Country (Study } \\
\text { Name) }\end{array}$ & $\begin{array}{l}\text { Study } \\
\text { Design }\end{array}$ & $\begin{array}{l}\text { Age } \\
\text { (Years) }\end{array}$ & Subjects & $\begin{array}{l}\text { Criteria for } \\
\text { Metabolic } \\
\text { Syndrome }\end{array}$ & Exposure Category & Adjustment Factors \\
\hline Kwon (2010) [50] & $\begin{array}{l}\text { Korea } \\
\text { (KNHANES III) }\end{array}$ & $\begin{array}{l}\text { Cross- } \\
\text { sectional }\end{array}$ & $\geq 19$ & $1066 / 4890$ & NCEP ATP III & $\begin{array}{l}\text { Milk } \\
\text { rarely (ref.), } \\
\leq 1 / \text { week, } 2-6 / \text { week, } \\
\geq 1 \text { /day }\end{array}$ & $\begin{array}{l}\text { Age, sex, BMI, education, } \\
\text { smoking, PA, alcohol, energy, } \\
\text { and fiber intake }\end{array}$ \\
\hline Jung (2011) [18] & $\begin{array}{l}\text { Korea (Bundang } \\
\text { Jesaeng General } \\
\text { Hospital, BJGH) }\end{array}$ & $\begin{array}{l}\text { Cross- } \\
\text { sectional }\end{array}$ & $30-59$ & $142 / 596$ & NCEP ATP III & $\begin{array}{l}\text { Dairy products } \\
\text { Q1 (ref.), Q2, Q3, Q4 }\end{array}$ & Age, sex, energy intake \\
\hline $\begin{array}{l}\text { Mosley } \\
\text { (2013) [19] }\end{array}$ & $\begin{array}{l}\text { Mexico (2009 UP } \\
\text { AMIGOS cohort) }\end{array}$ & $\begin{array}{l}\text { Cross- } \\
\text { sectional }\end{array}$ & $18-25$ & 339 & JIS & $\begin{array}{l}\text { Dairy products } \\
<3 \text { (ref.), } \geq 3 \\
\text { servings/day } \\
\text { Whole milk } \\
<7 \text { (ref.), } \geq 7 \\
\text { servings/week } \\
\text { Cheese } \\
<7 \text { (ref.), } \geq 7 \\
\text { servings/week }\end{array}$ & $\begin{array}{l}\text { Age, sex, total calorie, family } \\
\text { history, and PA }\end{array}$ \\
\hline Kim (2013) [51] & $\begin{array}{l}\text { Korea } \\
\text { (KNHANES V-1) }\end{array}$ & $\begin{array}{l}\text { Cross- } \\
\text { sectional }\end{array}$ & $\geq 19$ & 4862 & JIS & 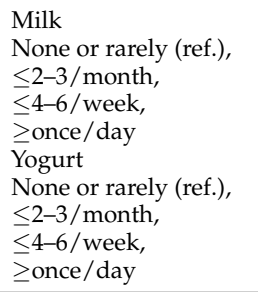 & $\begin{array}{l}\text { Age, sex, education, income, } \\
\text { smoking, BMI, alcohol, PA, } \\
\text { energy, fat, Ca, and fibre intake }\end{array}$ \\
\hline $\begin{array}{l}\text { Sadeghi } \\
(2014) \text { [53] }\end{array}$ & $\begin{array}{l}\text { Iran (Isfahan } \\
\text { Healthy Heart } \\
\text { Program, IHHP) }\end{array}$ & $\begin{array}{l}\text { Cross- } \\
\text { sectional }\end{array}$ & $\begin{array}{l}37.84 \\
39.08\end{array}$ & 1752 & $\begin{array}{l}3 \text { or more factor: } \\
\text { FBS }>126 \mathrm{mg} / \mathrm{dl} \\
\text { or waist }>102 \mathrm{~cm} \\
\text { for men and }>85 \\
\mathrm{~cm} \text { for women or } \\
\mathrm{TG}>150 \mathrm{mg} / \mathrm{dl} \\
\text { or } \mathrm{HLD}<40 \\
\mathrm{mg} / \mathrm{dl} \text { for men } \\
\text { and }<50 \mathrm{mg} / \mathrm{dl} \\
\text { for women or } \\
\text { systolic } \mathrm{BP}>130 \\
\text { mmHg and } \\
\text { diastolic }>85 \\
\text { mmHg. }\end{array}$ & $\begin{array}{l}\text { Cheese } \\
<7 \text { (ref.), } \geq 7 \\
\text { times/week }\end{array}$ & $\begin{array}{l}\text { Age, sex, dietary intake, PA, } \\
\text { BMI }\end{array}$ \\
\hline Kai (2014) [52] & $\begin{array}{l}\text { France (The } \\
\text { 2005-2007 } \\
\text { MONA LISA } \\
\text { multicentre } \\
\text { cross-sectional } \\
\text { population } \\
\text { survey) }\end{array}$ & $\begin{array}{l}\text { Cross- } \\
\text { sectional }\end{array}$ & $35-64$ & 3078 & JIS & $\begin{array}{l}\text { Dairy products } \\
0-13.2 \text { (ref.), } 13.3-23.1, \\
23.2-36.3,36.4-266.0 \\
\text { g/1000 kJ }\end{array}$ & $\begin{array}{l}\text { Age, sex, region, education, PA, } \\
\text { alcohol, smoking, diet, total } \\
\text { energy intake and Programme } \\
\text { National Nutrition } \\
\text { Sante'-Global Score }\end{array}$ \\
\hline $\begin{array}{l}\text { Martins } \\
(2015)[54]\end{array}$ & $\begin{array}{l}\text { Brazil (Perinatal } \\
\text { Health in } \\
\text { Ribeirao Preto, } \\
\text { Sao Paulo, } \\
\text { Brazil) }\end{array}$ & $\begin{array}{l}\text { Cross- } \\
\text { sectional }\end{array}$ & $23-25$ & $242 / 2031$ & $\begin{array}{l}\text { IDF } \\
\text { JIS }\end{array}$ & $\begin{array}{l}\text { Dairy products } \\
0.0-0.6 \text { (ref.), } 0.7-1.2, \\
1.3-1.7,1.8-2.6, \\
2.7-14.2 \text { portions /d }\end{array}$ & $\begin{array}{l}\text { Age, sex, smoking, alcohol, PA, } \\
\text { calorie intake, schooling and } \\
\text { marital status, carbohydrate, } \\
\text { protein intake, fat, bread and } \\
\text { cereal, vegetables, fruits, meats, } \\
\text { sugar and fats, Ca }\end{array}$ \\
\hline $\begin{array}{l}\text { Strand } \\
(2015)[20]\end{array}$ & $\begin{array}{l}\text { China (North } \\
\text { China Urban } \\
\text { Middle-Aged } \\
\text { Population) }\end{array}$ & $\begin{array}{l}\text { Cross- } \\
\text { sectional }\end{array}$ & $44,48,52$ & 793 & NCEP ATP III & $\begin{array}{l}\text { Milk } \\
\text { Rarely (ref.), } \\
\text { sometimes, often }\end{array}$ & $\begin{array}{l}\text { Age, sex, education, exercise, } \\
\text { alcohol, smoking, chronic } \\
\text { disease knowledge score, } \\
\text { family history }\end{array}$ \\
\hline $\begin{array}{l}\text { Drehmer } \\
(2016)[55]\end{array}$ & $\begin{array}{l}\text { Brazil (Brazilian } \\
\text { Longitudinal } \\
\text { study of Adult } \\
\text { Health, } \\
\text { ELSA-Brasil) }\end{array}$ & $\begin{array}{l}\text { Cross- } \\
\text { sectional }\end{array}$ & $35-74$ & 9835 & JIS & $\begin{array}{l}\text { Dairy products } \\
<1 \text { (ref.), } 1-2,>2-4,>4 \\
\text { servings / day }\end{array}$ & $\begin{array}{l}\text { Age, sex, race, alcohol, PA, } \\
\text { education, occupational status, } \\
\text { family income, study center, } \\
\text { menopausal status, family } \\
\text { history, smoking, and calorie } \\
\text { intake, nondairy food groups }\end{array}$ \\
\hline Falahi (2016) [56] & Iran & $\begin{array}{l}\text { Cross- } \\
\text { sectional }\end{array}$ & $18-75$ & $282 / 973$ & JIS & $\begin{array}{l}\text { Yogurt } \\
\text { Across mean } \\
\text { consumption }\end{array}$ & $\begin{array}{l}\text { Age, sex, smoking, PA, history } \\
\text { of diabetes and heart disease, } \\
\text { BMI, energy intake milk and } \\
\text { cheese intake }\end{array}$ \\
\hline
\end{tabular}


Table 3. cont.

\begin{tabular}{|c|c|c|c|c|c|c|c|}
\hline $\begin{array}{l}\text { First Author } \\
\text { (Year) }\end{array}$ & $\begin{array}{l}\text { Country (Study } \\
\text { Name) }\end{array}$ & $\begin{array}{c}\text { Study } \\
\text { Design }\end{array}$ & $\begin{array}{l}\text { Age } \\
\text { (Years) }\end{array}$ & Subjects & $\begin{array}{l}\text { Criteria for } \\
\text { Metabolic } \\
\text { Syndrome }\end{array}$ & Exposure Category & Adjustment Factors \\
\hline Shin (2017) [60] & $\begin{array}{l}\text { Korea (the } \\
\text { Health } \\
\text { Examinees study, } \\
\text { HEXA) }\end{array}$ & $\begin{array}{l}\text { Cross- } \\
\text { sectional }\end{array}$ & $40-69$ & $34,039 / 130,420$ & NCEP ATP III & $\begin{array}{l}\text { Milk } \\
\text { M: Non or Rarely } \\
\text { (ref.), } \leq 2 / \text { week, } \\
\text { 3-6/week, } \geq 1 / \text { day } \\
\text { F: Non or Rarely (ref.), } \\
\leq 2 / \text { week, 3-6/week, } \\
\text { 1/day, } \geq 2 / \text { day } \\
\text { Yogurt } \\
\text { The lowest (ref.), the } \\
\text { highest }\end{array}$ & $\begin{array}{l}\text { Age, BMI, recruitment site, } \\
\text { education, smoking, alcohol, } \\
\text { regular exercisers, and total } \\
\text { energy intake. }\end{array}$ \\
\hline Guo (2017) [57] & China & $\begin{array}{l}\text { Cross- } \\
\text { sectional }\end{array}$ & $\geq 18$ & $4305 / 15,020$ & JIS & $\begin{array}{l}\text { Milk } \\
\text { no or }<0.5 \text { (ref.), } \\
0.5-1.5, \geq 1.5 \mathrm{~L} / \text { week }\end{array}$ & $\begin{array}{l}\text { Age, education, minority, } \\
\text { vegetables, fresh meat, } \\
\text { drinking and smoking }\end{array}$ \\
\hline Kim (2017) [58] & $\begin{array}{l}\text { Korea } \\
\text { (KNHANES } \\
\text { IV-2,3, V-1,2) }\end{array}$ & $\begin{array}{l}\text { Cross- } \\
\text { sectional }\end{array}$ & $30-64$ & $3143 / 11,029$ & NCEP ATP III & $\begin{array}{l}\text { Milk } \\
\text { Q1 (ref.), Q2, Q3 }\end{array}$ & $\begin{array}{l}\text { Age, sex, total energy intake, } \\
\text { diet modification, and } \\
\text { education level }\end{array}$ \\
\hline $\begin{array}{l}\text { Mahanta } \\
(2017)[59]\end{array}$ & India & $\begin{array}{l}\text { Cross- } \\
\text { sectional }\end{array}$ & $20-60$ & $1606 / 3372$ & NCEP ATP III & $\begin{array}{l}\text { Dairy products } \\
<3 \text { (ref.), } \geq 3 \\
\text { times/week }\end{array}$ & $\begin{array}{l}\text { Age, religion, education, } \\
\text { occupation, car, motorcycle, } \\
\text { television, other land/property, } \\
\text { computer, family history } \\
\text { (hypertension, diabetes), } \\
\text { tobacco user, consumed } \\
\text { alcohol, financial stress, felt } \\
\text { stress in last year, active at } \\
\text { work, meat, fish, egg, high } \\
\text { energy food, desserts/sweet, } \\
\text { nuts/seeds, and past } 12 \\
\text { months, was ever you felt sad, } \\
\text { blue or depressed for } 2 \text { weeks } \\
\text { or more in a row }\end{array}$ \\
\hline $\begin{array}{l}\text { Chang } \\
(2019)[21]\end{array}$ & Taiwan & $\begin{array}{l}\text { Cross- } \\
\text { sectional }\end{array}$ & $\geq 20$ & $366 / 1066$ & NCEP ATP III & $\begin{array}{l}\text { Dairy products } \\
\text { Seldom(ref.), often }\end{array}$ & $\begin{array}{l}\text { Age, education, marital status } \\
\text { and employment }\end{array}$ \\
\hline $\begin{array}{l}\text { Bhavadharini } \\
\text { (2020) [61] }\end{array}$ & $\begin{array}{l}\text { Multinational } \\
\text { (Prospective } \\
\text { Urban Rural } \\
\text { Epidemiological } \\
\text { Study, PURE) }\end{array}$ & $\begin{array}{l}\text { Cross- } \\
\text { sectional }\end{array}$ & $35-70$ & 112,922 & JIS & $\begin{array}{l}\text { Dairy products } \\
0 \text { (ref.), }<1,1-2,>2 \\
\text { servings/day } \\
\text { Milk } \\
0 \text { (ref.), } 0-0.5,0.5-1,>1 \\
\text { servings/day } \\
\text { Yogurt } \\
\text { 0(ref.), } 0-0.5,0.5-1,>1 \\
\text { servings / day } \\
\text { Cheese } \\
\text { 0(ref.), } 0-0.5,0.5-1,>1 \\
\text { servings/day }\end{array}$ & $\begin{array}{l}\text { Age, sex, smoking, energy } \\
\text { intake, education, location, PA, } \\
\text { fruit and vegetable intake, } \\
\text { percent energy from } \\
\text { carbohydrate, and study center } \\
\text { as random effect }\end{array}$ \\
\hline $\begin{array}{l}\text { Pasdar } \\
(2020)[64]\end{array}$ & Iran & $\begin{array}{l}\text { Cross- } \\
\text { sectional }\end{array}$ & $30-65$ & $52 / 112$ & IDF & $\begin{array}{l}\text { Dairy products } \\
<3 \text { (ref.), } 3-5,>5 \\
\text { times/day }\end{array}$ & Age, BMI, and PA \\
\hline $\begin{array}{l}\text { Hidayat } \\
(2020)[62]\end{array}$ & China & $\begin{array}{l}\text { Cross- } \\
\text { sectional }\end{array}$ & $\geq 18$ & $2387 / 5149$ & JIS & $\begin{array}{l}\text { Milk } \\
\text { Non-consumer (ref.), } \\
\text { consumer }\end{array}$ & $\begin{array}{l}\text { Age, sex, smoking, alcohol, } \\
\text { BMI, PA, education, television } \\
\text { watching duration, sleep } \\
\text { duration, and consumption of } \\
\text { fish, red meat, poultry, } \\
\text { vegetables, fruits, nut, soya } \\
\text { and salted vegetables }\end{array}$ \\
\hline $\begin{array}{l}\text { Mohammadifard } \\
\text { (2020) [63] }\end{array}$ & $\begin{array}{l}\text { Iran (Isfahan } \\
\text { Healthy Heart } \\
\text { Program, IHHP) }\end{array}$ & $\begin{array}{l}\text { Cross- } \\
\text { sectional }\end{array}$ & $\geq 19$ & 9553 & NCEP ATP III & $\begin{array}{l}\text { Dairy products } \\
<12 \text { (ref.), } 12-16, \\
>16-21,>21 \\
\text { times/week }\end{array}$ & $\begin{array}{l}\text { Age, sex, urbanization, } \\
\text { educational level education, } \\
\text { BMI, PA, history of CVD, and } \\
\text { dietary factors }\end{array}$ \\
\hline Jin (2020) [65] & $\begin{array}{l}\text { Korea } \\
\text { (KNHANES VI, } \\
\text { VII) }\end{array}$ & $\begin{array}{l}\text { Cross- } \\
\text { sectional }\end{array}$ & $\geq 19$ & $6325 / 23,319$ & NCEP ATP III & $\begin{array}{l}\text { Dairy products } \\
0 \text { (ref.), } 0<\text { to }<1, \geq 1 \\
\text { serving/day }\end{array}$ & $\begin{array}{l}\text { Age, sex, smoking, alcohol, } \\
\text { BMI, education, household } \\
\text { income, PA, and total energy }\end{array}$ \\
\hline KNHANES $^{1}$ & $\begin{array}{l}\text { Korea } \\
\text { (KNHANES VI, } \\
\text { VII) }\end{array}$ & $\begin{array}{l}\text { Cross- } \\
\text { sectional }\end{array}$ & $\geq 19$ & $6325 / 23,319$ & NCEP ATP III & $\begin{array}{l}\text { Milk } \\
0 \text { (ref.), } 0<\text { to }<1, \geq 1 \\
\text { serving/day } \\
\text { Yogurt } \\
0 \text { (ref.), } 0<\text { to }<1, \geq 1 \\
\text { serving/day }\end{array}$ & $\begin{array}{l}\text { Age, sex, smoking, alcohol, } \\
\text { BMI, education, household } \\
\text { income, PA, and total energy }\end{array}$ \\
\hline
\end{tabular}

Abbreviations: BP, blood pressure; BMI, body mass index; PA, physical activity; NCEP ATP III, National Cholesterol Education Program Adult Treatment Panel III; IDF, International Diabetes Federation; ALT, alanine aminotransferase; JIS, Joint Interim Statement; WHO, World Health Organization; KNHANES, Korea National Health and Nutrition Examination Survey; M, male; F, female; CVD, Cardiovascular Disease. ${ }^{1}$ Data of the KNHANES analysis from the current paper. 


\subsubsection{Total Dairy Consumption and MetS}

Twenty-two studies investigated the association between the highest versus the lowest total dairy consumption and risk of MetS, and included 210,790 participants (Table 3). The pooled RR for all studies was 0.80 (95\% CI: 0.72-0.88) (Table 4), with some evidence of substantial heterogeneity $\left(I^{2}=82.0 \%, p<0.001\right)$. A similar inverse association was found in cohort and cross-sectional studies (Table 4, Figure S1a). By sex, the inverse association tended to be stronger in women than men. By geographic region, there was a significant inverse association in America, Asia, and Europe, while only one study from Oceania showed no significant association. Regarding the criteria of MetS, a significant inverse association was shown in NCEP ATP III, JIS, and other criteria but not in IDF criteria. Furthermore, the heterogeneity disappeared in JIS $\left(I^{2}=20.2 \%, p=0.28\right)$, IDF $\left(I^{2}=57.3 \%\right.$, $p=0.07)$, and other criteria $\left(I^{2}=31.3 \%, p=0.22\right)$ when stratified by the criteria. When we conducted a meta-regression analysis to assess the variations in risk estimates, however, there was no significant difference in the relationship between dairy consumption and risk of MetS with study design, sex, geographical region, or criteria ( $p_{\text {difference }}>0.5$ for all comparisons). For adjustment for confounders, an inverse association was found in studies adjusted for energy intake, but the difference in the pooled RRs with energy intake adjustment was not significant $\left(p_{\text {difference }}=0.28\right.$ ). In addition, there was no significant difference with other adjustment factors ( $p_{\text {difference }}>0.2$ for all comparisons). Six cohort studies $[22,35,38,40,41,43]$ were included in the dose-response analysis for total dairy consumption and incidence of MetS (Table 5). The pooled RR for $400 \mathrm{~g} /$ day increment of total dairy consumption was 0.71 (95\% CI: $0.59-0.85, I^{2}=72.4 \%, p=0.003$ ).

Table 4. Subgroup-specific pooled of pooled relative risks for dairy consumption and risk of metabolic syndrome.

\begin{tabular}{|c|c|c|c|}
\hline Subgroups & No. of Studies & Relative Risk (95\% CI) & $p_{\text {difference }}$ \\
\hline Dairy & 22 & $0.80(0.72-0.88)$ & \\
\hline \multicolumn{4}{|l|}{ Study design } \\
\hline Cohort & 8 & $0.75(0.65-0.87)$ & 0.53 \\
\hline Cross-sectional & 16 & $0.82(0.72-0.92)$ & \\
\hline \multicolumn{4}{|l|}{ Sex } \\
\hline Men & 8 & $0.77(0.62-0.95)$ & 0.66 \\
\hline Women & 7 & $0.72(0.59-0.88)$ & \\
\hline \multicolumn{4}{|l|}{ Geographical region } \\
\hline America & 7 & $0.83(0.69-0.99)$ & \\
\hline Asia & 8 & $0.78(0.63-0.96)$ & $0.85^{1}$ \\
\hline Europe & 5 & $0.85(0.78-0.93)$ & $0.99^{1}$ \\
\hline Oceania & 1 & $0.62(0.24-1.61)$ & $0.70^{1}$ \\
\hline \multicolumn{4}{|l|}{ Criteria } \\
\hline NCEP ATP III & 12 & $0.82(0.71-0.94)$ & \\
\hline JIS & 7 & $0.77(0.72-0.83)$ & $0.62^{2}$ \\
\hline IDF & 4 & $0.73(0.43-1.24)$ & $0.87^{2}$ \\
\hline Other & 3 & $0.76(0.60-0.95)$ & $0.55^{2}$ \\
\hline \multicolumn{4}{|c|}{ Adjustment for confounders } \\
\hline \multicolumn{4}{|c|}{ BMI } \\
\hline Yes & 8 & $0.75(0.66-0.86)$ & 0.41 \\
\hline No & 14 & $0.84(0.72-0.97)$ & \\
\hline \multicolumn{4}{|l|}{ Energy intake } \\
\hline Yes & 16 & $0.76(0.69-0.85)$ & 0.28 \\
\hline No & 6 & $0.90(0.69-1.16)$ & \\
\hline \multicolumn{4}{|l|}{ Alcohol } \\
\hline Yes & 12 & $0.84(0.72-0.99)$ & 0.28 \\
\hline No & 10 & $0.77(0.70-0.86)$ & \\
\hline \multicolumn{4}{|l|}{ Smoking } \\
\hline Yes & 15 & $0.81(0.73-0.90)$ & 0.37 \\
\hline No & 7 & $0.71(0.56-0.91)$ & \\
\hline \multicolumn{4}{|l|}{ Physical activity } \\
\hline Yes & 19 & $0.82(0.74-0.91)$ & 0.31 \\
\hline No & 3 & $0.66(0.50-0.87)$ & \\
\hline
\end{tabular}


Table 4. cont.

\begin{tabular}{|c|c|c|c|}
\hline Subgroups & No. of Studies & Relative Risk (95\% CI) & $p_{\text {difference }}$ \\
\hline Milk & 20 & $0.83(0.78-0.89)$ & \\
\hline \multicolumn{4}{|l|}{ Study design } \\
\hline Cohort & 7 & $0.83(0.72-0.97)$ & 0.94 \\
\hline Cross-sectional & 13 & $0.83(0.77-0.90)$ & \\
\hline \multicolumn{4}{|l|}{ Sex } \\
\hline Men & 7 & $0.83(0.75-0.92)$ & 0.70 \\
\hline Women & 7 & $0.79(0.69-0.90)$ & \\
\hline \multicolumn{4}{|l|}{ Geographical region } \\
\hline America & 6 & $0.86(0.78-0.95)$ & \\
\hline Asia & 10 & $0.80(0.72-0.89)$ & $0.65^{3}$ \\
\hline Europe & 3 & $0.87(0.45-1.71)$ & $0.72^{3}$ \\
\hline \multicolumn{4}{|l|}{ Criteria } \\
\hline NCEP ATP III & 11 & $0.84(0.77-0.92)$ & \\
\hline JIS & 7 & $0.84(0.77-0.93)$ & $0.88^{4}$ \\
\hline IDF & 1 & $0.79(0.59-1.07)$ & $0.76^{4}$ \\
\hline Other & 3 & $0.83(0.41-1.67)$ & $0.98^{4}$ \\
\hline \multicolumn{4}{|c|}{ Adjustment for confounders } \\
\hline \multicolumn{4}{|c|}{ BMI } \\
\hline Yes & 8 & $0.81(0.73-0.89)$ & 0.53 \\
\hline No & 12 & $0.86(0.78-0.95)$ & \\
\hline \multicolumn{4}{|l|}{ Energy intake } \\
\hline Yes & 13 & $0.83(0.78-0.89)$ & 0.89 \\
\hline No & 7 & $0.83(0.66-1.05)$ & \\
\hline \multicolumn{4}{|l|}{ Alcohol } \\
\hline Yes & 14 & $0.82(0.75-0.88)$ & 0.47 \\
\hline No & 6 & $0.88(0.75-1.04)$ & \\
\hline \multicolumn{4}{|l|}{ Smoking } \\
\hline Yes & 16 & $0.81(0.76-0.88)$ & 0.27 \\
\hline No & 4 & $0.95(0.75-1.20)$ & \\
\hline \multicolumn{4}{|l|}{ Physical activity } \\
\hline Yes & 14 & $0.83(0.77-0.90)$ & 0.88 \\
\hline No & 6 & $0.84(0.71-0.99)$ & \\
\hline Yogurt & 12 & $0.89(0.83-0.95)$ & \\
\hline \multicolumn{4}{|l|}{ Study design } \\
\hline Cohort & 6 & $0.84(0.71-0.98)$ & 0.27 \\
\hline \multirow{2}{*}{\multicolumn{4}{|c|}{ Sex }} \\
\hline & & & \\
\hline Men & 4 & $0.86(0.72-1.02)$ & 0.71 \\
\hline Women & 4 & $0.91(0.81-1.02)$ & \\
\hline \multicolumn{4}{|l|}{ Geographical region } \\
\hline America & 3 & $0.71(0.42-1.22)$ & \\
\hline Asia & 6 & $0.91(0.84-0.998)$ & $0.70^{5}$ \\
\hline Europe & 2 & $0.78(0.67-0.91)$ & $0.70^{5}$ \\
\hline \multicolumn{4}{|l|}{ Criteria } \\
\hline NCEP ATP III & 4 & $0.81(0.68-0.97)$ & \\
\hline JIS & 6 & $0.89(0.81-0.98)$ & $0.40^{6}$ \\
\hline IDF & 1 & $1.00(0.93-1.06)$ & $0.09^{6}$ \\
\hline Other & 1 & $0.58(0.20-1.67)$ & $0.56^{6}$ \\
\hline \multicolumn{4}{|c|}{ Adjustment for confounders } \\
\hline BMI & & & \\
\hline Yes & 8 & $0.89(0.82-0.97)$ & 0.84 \\
\hline No & 4 & $0.89(0.81-0.97)$ & \\
\hline Energy intake & & & \\
\hline Yes & 11 & $0.90(0.85-0.97)$ & 0.32 \\
\hline No & 1 & $0.77(0.65-0.91)$ & \\
\hline Alcohol & & & \\
\hline Yes & 9 & $0.86(0.77-0.95)$ & 0.30 \\
\hline No & 3 & $0.94(0.85-1.03)$ & \\
\hline Smoking & & & \\
\hline Yes & 11 & $0.90(0.84-0.96)$ & 0.12 \\
\hline No & 1 & $0.42(0.18-0.99)$ & \\
\hline Physical activity & & & \\
\hline Yes & 10 & $0.87(0.81-0.94)$ & 0.27 \\
\hline No & 2 & $0.96(0.87-1.06)$ & \\
\hline
\end{tabular}


Table 4. cont.

\begin{tabular}{|c|c|c|c|}
\hline Subgroups & No. of Studies & Relative Risk (95\% CI) & $p_{\text {difference }}$ \\
\hline Cheese & 8 & $0.98(0.86-1.11)$ & \\
\hline \multicolumn{4}{|l|}{ Study design } \\
\hline Cohort & 4 & $1.03(0.87-1.22)$ & \multirow[t]{2}{*}{0.43} \\
\hline Cross-sectional & 4 & $0.91(0.74-1.14)$ & \\
\hline \multicolumn{4}{|l|}{ Geographical region } \\
\hline America & 3 & $1.07(0.93-1.25)$ & \multirow{3}{*}{$\begin{array}{c}0.66^{7} \\
0.996^{7}\end{array}$} \\
\hline Asia & 2 & $0.92(0.71-1.20)$ & \\
\hline Europe & 2 & $1.03(0.65-1.64)$ & \\
\hline \multicolumn{4}{|l|}{ Criteria } \\
\hline NCEP ATP III & 3 & $1.00(0.83-1.20)$ & \multirow{4}{*}{$\begin{array}{l}0.91^{8} \\
0.61^{8} \\
0.42^{8}\end{array}$} \\
\hline JIS & 4 & $1.01(0.79-1.29)$ & \\
\hline IDF & 1 & $0.88(0.77-1.00)$ & \\
\hline Other & 1 & $0.81(0.70-0.93)$ & \\
\hline \multicolumn{4}{|c|}{ Adjustment for confounders } \\
\hline \multicolumn{4}{|c|}{ BMI } \\
\hline Yes & 4 & $0.98(0.78-1.21)$ & \multirow[t]{2}{*}{0.97} \\
\hline No & 4 & $0.99(0.82-1.18)$ & \\
\hline \multicolumn{4}{|l|}{ Energy intake } \\
\hline Yes & 5 & $1.00(0.87-1.16)$ & \multirow[t]{2}{*}{0.75} \\
\hline No & 3 & $0.95(0.71-1.27)$ & \\
\hline \multicolumn{4}{|l|}{ Alcohol } \\
\hline Yes & 3 & $1.15(1.01-1.30)$ & \multirow[t]{2}{*}{0.02} \\
\hline No & 5 & $0.87(0.79-0.96)$ & \\
\hline \multicolumn{4}{|l|}{ Smoking } \\
\hline Yes & 5 & $0.99(0.86-1.14)$ & \multirow[t]{2}{*}{0.75} \\
\hline No & 3 & $0.92(0.66-1.29)$ & \\
\hline \multicolumn{4}{|l|}{ Physical activity } \\
\hline Yes & 6 & $0.95(0.80-1.14)$ & \multirow[t]{2}{*}{0.63} \\
\hline No & 2 & $1.03(0.95-1.12)$ & \\
\hline
\end{tabular}

Abbreviations: NCEP ATP III, National Cholesterol Education Program Adult Treatment Panel III; IDF, International Diabetes Federation; JIS, Joint Interim Statement. ${ }^{1} p$ value for difference in RRs of dairy consumption for Asia versus America, Europe versus America, and Oceania versus America. ${ }^{2} p$ value for difference in RRs of dairy consumption for JIS criteria versus NCEP ATP III criteria, IDF criteria versus NCEP ATP III criteria, and other criteria versus NCEP ATP III criteria. ${ }^{3} p$ value for difference in RRs of milk consumption for Asia versus America and Europe versus America. ${ }^{4} p$ value for difference in RRs of milk consumption for JIS criteria versus NCEP ATP III criteria, IDF criteria versus NCEP ATP III criteria, and other criteria versus NCEP ATP III criteria. ${ }^{5} p$ value for difference in RRs of yogurt consumption for Asia versus America and Europe versus America. ${ }^{6} p$ value for difference in RRs of yogurt consumption for JIS criteria versus NCEP ATP III criteria, IDF criteria versus NCEP ATP III criteria, and other criteria versus NCEP ATP III criteria. ${ }^{7} p$ value for difference in RRs of cheese consumption for Asia versus America and Europe versus America. ${ }^{8} p$ value for difference in RRs of cheese consumption for JIS criteria versus NCEP ATP III criteria, IDF criteria versus NCEP ATP III criteria, and other criteria versus NCEP ATP III criteria. Abbreviations: NCEP ATP III, National Cholesterol Education Program Adult Treatment Panel III; JIS, Joint Interim Statement IDF; International Diabetes Federation.

Table 5. Pooled RRs of dairy consumption and metabolic syndrome incidence from dose-response meta-analysis.

\begin{tabular}{ccccc}
\hline & No of Studies & Dose & Relative Risk (95\% CI) & Heterogeneity \\
\hline Total airy & 6 & $400 \mathrm{~g} /$ day & $0.71(0.59-0.85)$ & $I^{2}=72.4 \%, p=0.003$ \\
Milk & 5 & $200 \mathrm{~g} /$ day & $0.85(0.79-0.93)$ & $I^{2}=51.8 \%, p=0.08$ \\
Yogurt & 5 & $200 \mathrm{~g} /$ day & $0.63(0.53-0.75)$ & $I^{2}=0.3 \%, p=0.40$ \\
Cheese & 3 & $50 \mathrm{~g} /$ day & $0.99(0.73-1.35)$ & $I^{2}=86.2 \%, p=0.001$ \\
\hline
\end{tabular}

\subsubsection{Milk Consumption and MetS}

Twenty studies investigated the association between the highest versus the lowest milk consumption and risk of MetS, and included 341,426 participants (Table 3). The pooled RR for all studies was 0.83 (95\% CI: 0.78-0.89) (Table 4), with evidence of substantial heterogeneity $\left(I^{2}=72.5 \%, p<0.001\right)$. Furthermore, in the stratified analysis by study design, we found a similar inverse association in cohort and cross-sectional studies (Table 4 , 
Figure S1b). By sex, we found a significant inverse association in men and women, and no significant difference was found $\left(p_{\text {difference }}=0.70\right)$. By geographic region, there was a significant inverse association in America and Asia, while there was no significant association in Europe. By criteria of MetS, significant inverse associations were shown in NCEP ATP III and JIS criteria, while no significant association was found in IDF and other criteria. In the meta-regression analysis, however, the pooled RRs were not significantly different with criteria of MetS ( $p_{\text {difference }}$ for JIS, IDF, or other criteria versus NCEP ATP III criteria $=0.88,0.76$, and 0.98 , respectively). By adjustment factors, we found no significant difference with BMI, energy intake, alcohol, smoking, or physical activity $\left(p_{\text {difference }}>0.2\right.$ for all comparisons). Five cohort studies $[22,34,41,43,44]$ were included in the dose-response analysis for milk consumption and incidence of MetS (Table 5). A $200 \mathrm{~g} /$ day increment of milk consumption was associated with $15 \%$ decrease incidence of MetS (RR: $0.85 ; 95 \%$ CI: $0.79-0.93)$, with no significant heterogeneity $\left(I^{2}=51.8 \%, p=0.08\right)$.

\subsubsection{Yogurt Consumption and MetS}

Twelve studies investigated the association between the highest versus the lowest yogurt consumption and risk of MetS, and included 300,600 participants (Table 3). The pooled RR for all studies was 0.89 (95\% CI: 0.83-0.95), with some evidence of heterogeneity $\left(I^{2}=69.1 \%, p<0.001\right)$. By study design, we found a similar inverse association in cohort and cross-sectional studies (Table 4, Figure S1c). By geographic region, a significant inverse association was found in Asia and Europe, while there was no significant association in America. Furthermore, the heterogeneity disappeared in America $\left(I^{2}=51.1 \%, p=0.13\right)$ and Europe $\left(I^{2}=0 \%, p=0.65\right)$ when we stratified by the region. By Met $S$ criteria, a significant inverse association was found in the NCEP ATP III and JIS criteria, while only one study was included in IDF and other criteria. There was no significant difference in geographic region or criteria ( $p_{\text {difference }}>0.05$ for all comparisons). Regarding adjustment for confounders, we found no significant difference with BMI, energy intake, alcohol, smoking, or physical activity ( $p_{\text {difference }}>0.1$ for all comparisons). Five cohort studies [22,41-44] were included in the dose-response analysis for yogurt consumption and incidence of MetS (Table 5). A $200 \mathrm{~g} /$ day increment of yogurt intake was associated with 37\% decrease incidence of MetS (RR: $0.63 ; 95 \%$ CI: 0.53-0.75), with no significant heterogeneity $\left(I^{2}=0.3 \%, p=0.40\right)$.

\subsubsection{Cheese Consumption and MetS}

Eight studies investigated the association between the highest versus the lowest total dairy consumption and risk of MetS, and included 129,822 participants (Table 3). The pooled RR for all studies was 0.98 (95\% CI: 0.86-1.11), with evidence of substantial heterogeneity $\left(I^{2}=85.6 \%, p<0.001\right)$. Similarly, there was no significant association both in cohort and cross-sectional studies (Table 4, Figure S1d). Based on the results of metaregression analyses, there was no significant difference with study design, geographic region, or MetS criteria ( $p_{\text {difference }}>0.4$ for all comparisons). By adjustment for confounders, we found a significant positive association in studies that had adjusted for alcohol drinking, and the result of meta-regression analysis showed a significant difference $\left(p_{\text {difference }}=0.02\right)$. Three cohort studies $[38,41,44]$ were included in the dose-response analysis for cheese intake and incidence of MetS (Table 5). The pooled RR for $50 \mathrm{~g} /$ day increment of cheese consumption was 0.99 (95\% CI: 0.73-1.35, $\left.I^{2}=86.2 \%, p=0.001\right)$.

\subsubsection{Publication Bias}

There was no evidence of publication bias with Begg's ( $p>0.1$ in all analysis) and Egger's test ( $p>0.05$ in all analysis) of risk of MetS for total dairy, milk, yogurt, and cheese consumption.

\section{Discussion}

In the present meta-analysis, we found significant inverse associations with the risk of MetS for the highest vs. lowest categories of total dairy, milk, and yogurt consumption, 
while cheese consumption showed no significant association overall. People in the highest category of total dairy, milk, and yogurt consumption had a decreased risk of MetS of $20 \%$, $17 \%$, and $11 \%$, respectively, compared with those in the lowest intake category. The results of dose-response analysis also supported the associations.

We conducted several subgroup analyses in the present study. Published previous two meta-analyses conducted a subgroup analysis depending on the study design [6,7], and one meta-analysis conducted a subgroup analysis of total dairy and milk intake [8]. In addition, the most recent meta-analysis included only prospective cohort studies, and no subgroup analysis was performed [9]. We performed an analysis stratified by study design, and found no significant difference in study design. Thus, we included both cohort and cross-sectional studies, and conducted subgroup analyses for total dairy products, milk, yogurt, and cheese consumption. Moreover, we included more recent two cohort studies (three articles) $[23,24,44]$ and 11 cross-sectional studies (11 articles and the current results of the KNHANES data) [21,56-65] including the first large multinational studies [61]. In the present meta-analysis, we not only performed stratified analyzes by geographic region and adjustment for confounders like the previous meta-analyses $[7,8]$ but also performed analyses stratified by sex and MetS criteria.

Some evidence of heterogeneity among the studies was found in the meta-analysis of dairy consumption and the risk of MetS. The observed heterogeneity tended to disappear when stratified by geographic region or MetS criteria. According to the region, the dairy products consumption varies greatly. Europe is the region with the highest consumption of dairy products, while the intake of dairy products in Asia is much lower than that of European and American countries [66]. The result of KNHANES data in the current study also reflects this trend. More than three-quarters of people did not consume dairy products. In addition, the composition of dairy products varies according to different regions. Europe and the United States are both countries with high consumption of dairy products, but the United States consumes a higher proportion of processed dairy products than Europe [67]. There are more than one diagnostic criteria for MetS. Although the general standards are similar, they are not the same. According to the IDF definition, for a person to be diagnosed with MetS, they must have abdominal obesity and any two of the four factors [2]. For the JIS criteria, people with any three of five risk factors are diagnosed with MetS and there are standard recommended waist circumference thresholds for abdominal obesity by region [1]. The NCEP ATP III criteria requires at least three of the five components for the diagnosis of MetS [13], and several studies combined their region standard of waist circumference thresholds and made a modified NCEP ATP III criteria. The use of different criteria for MetS may lead to heterogeneity of the study results.

In the current analysis of KNHANES data, the study showed that consumption of milk was inversely associated with the prevalence of MetS in Korean elderly, but not in adults. Compared with adults, low-fat or skimmed milk accounts for a higher proportion of the total milk intake of the elderly. The previous cohort study reported that low-fat milk intake decreased the risk of MetS in the elderly, whereas whole-fat milk intake had no significant association [41]. Moreover, in the result of additional analysis of MetS components, milk consumption was associated with low HDL-cholesterol in elderly women. The percentage of total energy from carbohydrates in elderly women was extremely high, even exceeding the KDRI standard [12], and this tendency was more serious in non-consumers. The previous study reported that high carbohydrate intake were significantly associated with low HDL-cholesterol levels in women [68]. Although there was no significant association between milk consumption and risk of MetS in adults, milk consumption was associated with hypertriglyceridemia in adult men, and a previous study supported this association [69]. Regarding the consumption of yogurt, although the yogurt intake in adult women and elderly men was inversely associated with hyperglycemia, there was not significantly associated with the prevalence of MetS. This result may be because the type of yogurt consumed in Korea is different from traditional yogurts such as Greek yogurt or non-curd yogurt in Western countries [60]. In Korea, the most popular 
yogurt contains a lot of sugar [60]. Several previous studies have shown that consumption of fructose may cause type 2 diabetes and cardiovascular disease [70], but there was no study to explain the effect of adding sugar to yogurt on metabolism. On the other hand, although people who consumed higher levels of yogurt had higher calcium intake than non-consumers, the difference was not as extreme as the milk consumption.

The several potential mechanisms could explain the beneficial effect of dairy product consumption such as milk and yogurt on MetS. Dairy products contain several minerals such as calcium and potassium [71]. Several previous studies showed that increased intake of dairy products or calcium could lead to the prevention of weight gain or weight regain $[72,73]$. Increasing the dairy products or calcium intake can impair fat absorption by forming insoluble soaps, leading to weight and fat loss [71,74]. Moreover, some studies showed that an increase of calcium in the diet leads to an increase in fecal fat excretion [73]. Calcium combines with bile acids in the intestine to inhibit their absorption, and this is associated with reduced serum low-density lipoprotein cholesterol $[4,74,75]$. Insufficient calcium intake increases the level of 1,25-dihydroxycholecalciferol in plasma, which increases intracellular calcium concentration in adipocytes, resulting in the inhibition of lipolysis and FA synthase gene expression, thereby leading to the expansion of triglycerides storage in adipocytes [76]. By contrast, adequate calcium intake reduces the level of 1,25-dihydroxycholecalciferol in plasma, which leads to decrease in intracellular calcium in adipocytes, thereby reducing fatty acid (FA) synthase and increasing lipolytic activity $[4,76]$. In addition, calcium and potassium affect MetS through their effects on blood pressure [71]. Adequate dairy intake affects blood pressure control, which may be due to the ability of calcium and potassium to reduce sodium retention through several mechanisms [77]. 1,25-Dihydroxycholecalciferol stimulates calcium influx through vitamin D receptors, thereby promoting contraction and peripheral resistance [78]. Therefore, adequate calcium consumption will reduce 1,25-dihydroxycholecalciferol and thus lower blood pressure [78]. Potassium in the diet can reduce blood pressure by inhibiting proinflammatory events in vascular smooth muscle cells, reducing platelet aggregation and renal vascular resistance [71,79].

Dairy products also contain protein, which mainly includes casein and whey proteins. Hydrolyzed whey protein can inhibit the angiotensin-converting enzyme in vitro, thereby inducing the inhibition of angiotensin II [4,71]. In addition to the blood pressure regulating effect of angiotensin II, it can also up-regulate the expression of FA synthase gene, leading to adipocyte lipogenesis $[4,80,81]$. Thus, whey protein reduces endogenous fat, resulting in decreased plasma triglycerides, total cholesterol, and low-density lipoprotein (LDL)-cholesterol [71,82]. Dairy products contain also more than 400 different identified FAs and FA derivatives [5]. Among them, saturated FAs are the predominant FAs type in milk fat, accounting for about $64-73 \%$ of total FAs [5]. Butyric acid, one of the representative short-chain FAs included in dairy products, can induce positive changes in systemic metabolism [5]. Animal studies have shown that butyrate provided by a high-fat diet mitigates weight gain [83] and improves insulin sensitivity [84]. Similarly, the consumption of medium-chain FAs and long-chain FAs can also induce positive changes in metabolism [5]. Caprylic acid, a medium-chain FA, can beneficially influence glucose and energy homeostasis [5]. A diet rich in capric acid, another medium-chain FA, can lower total cholesterol and triglycerides $[85,86]$. Some research suggests that stearic acid (longchain FA) consumption can have a positive effect on whole-body energy metabolism [5]. However, a previous study reported that intake of long-chain FA had an increased risk of coronary heart disease [87].

The nutritional ingredients of dairy products vary according to each dairy product type. Yogurt is rich in probiotic bacteria that can beneficially improve the gut microbiota, which has a positive effect on weight control [88]. In addition, a previous meta-analysis of randomized trials showed that probiotic consumption may prevent and control hyperglycemia [89]. Cheese is a high-fat dairy product, and whey protein is lost during the cheese-making process. These differences may cause different effects between milk 
or yogurt and cheese. In addition, one cohort study reported cheese consumption was associated with an increased risk of MetS and explained that cheese has higher phosphorus content and energy density, and sodium than other dairy products [41]. However, a previous randomized trial showed that a high intake of cheese did not increase total or LDL cholesterol level and it was related to a slight decrease in cholesterol level in those participants who had MetS at baseline, compared with the control group of low cheese intake [90]. In the current study, cheese consumption was not significantly associated with the risk of MetS, and showed a slightly higher risk of MetS when limited to studies that had adjusted for alcohol consumption. Interestingly, all previous studies that cheese consumption had a positive effect on MetS have not adjusted for alcohol consumption [38,53,61], and a previous meta-analysis presented lower risk of MetS in light alcohol drinkers [91]. Thus, there is a lack of comprehensive explanation of the association between cheese intake and MetS, and more studies on cheese consumption are needed.

There are some strengths of the present meta-analysis. We performed several subgroup analyses with different types of dairy product. To the best of our knowledge, this is the first meta-analysis to assess the association between cheese consumption and MetS risk, and different types of dairy products have been individually stratified by sex, MetS criteria, and geographical region. The current study included the most recent prospective cohort and cross-sectional data, and the largest number of study subjects. Many studies included in the meta-analysis have adjusted for confounding factors such as age, sex, energy intake, alcohol consumption, smoking, or physical activity. In addition, the present study assessed a linear dose-response meta-analysis between dairy products consumption, such as total dairy, milk, yogurt, and cheese, and MetS incidence.

The present meta-analysis also has several limitations. First, this meta-analysis was based on observational studies and, therefore, possible residual or unmeasured confounding factors cannot be excluded from the results. Second, most of the studies included in current meta-analysis used self-reported food frequency questionnaires (FFQs) to assess the consumption of dairy products, and therefore, it is necessary to consider the possible measurement errors when recording information during the assessment. Third, the cut-offs for the highest and lowest consumption categories varied among the studies. To address this limitation, we conducted a dose-response meta-analysis as well. Fourth, in the KNHANES study, we used dietary data collected from $24 \mathrm{~h}$ dietary recall in order to include both adults and elderly, and thus, it may reflect the short-term intake of the participants rather than their usual intake.

\section{Conclusions}

In conclusion, the results of this meta-analysis of prospective cohort studies and cross-sectional studies suggest that total dairy products, milk, and yogurt consumption is associated with reduced risk of MetS, while cheese consumption is not significantly associated with the risk of MetS. The use of different diagnostic criteria for MetS and different geographical regions may have affected the heterogeneity of the results. Although our findings may provide deeper insights into the consumption of dairy products, further well-designed prospective cohort studies on different types of dairy products and different types of study populations are warranted. In addition, randomized clinical trials should be conducted to examine the association between different types of dairy products consumption and the risk of MetS to provide definitive evidence.

Supplementary Materials: The following are available online at https: / www.mdpi.com/article / 10.3390/nu13051574/s1, Table S1: General characteristics of the study participants according to milk and yogurt consumption in the Korean adult and elderly population, Table S2: Gender-specific general characteristics according to milk consumption in the Korean adult and elderly population, Table S3: Gender-specific general characteristics according to yogurt consumption in the Korean adult and elderly population, Table S4: Multivariable-adjusted odds ratio (ORs) and 95\% confidence intervals (CIs) for metabolic syndrome components according to milk consumption in Korean adult and elderly population, Table S5: Multivariable-adjusted odds ratio (ORs) and 95\% confidence inter- 
vals (CIs) for metabolic syndrome components according to yogurt consumption in Korean adult and elderly population. Figure S1: Forest plot of the observational studies for risk of metabolic syndrome.

Author Contributions: Conceptualization, Y.J. and S.J.; formal analysis, Y.J. and S.J.; investigation, Y.J. and S.J.; data curation, Y.J. and S.J.; writing-original draft preparation, S.J.; writing-review and editing, Y.J. All authors have read and agreed to the published version of the manuscript.

Funding: This research was funded by the National Research Foundation of Korea funded by the Korean government, grant number NRF-2018R1D1A1B07045353.

Institutional Review Board Statement: The study was conducted according to the guidelines of the Declaration of Helsinki, and approved by the Institutional Review Board of the Korea Centers for Disease Control and Prevention (2013-07CON-03-4C, 2013-12EXP-03-5C, 2018-01-03-P-A).

Informed Consent Statement: Informed consent was obtained from all subjects involved in the study.

Data Availability Statement: Data is contained within the article or supplementary material.

Conflicts of Interest: The authors declare no conflict of interest. The funders had no role in the design of the study; in the collection, analyses, or interpretation of data; in the writing of the manuscript; or in the decision to publish the results.

\section{References}

1. Alberti, K.G.; Eckel, R.H.; Grundy, S.M.; Zimmet, P.Z.; Cleeman, J.I.; Donato, K.A.; Fruchart, J.C.; James, W.P.; Loria, C.M.; Smith, S.C., Jr.; et al. Harmonizing the metabolic syndrome: A joint interim statement of the International Diabetes Federation Task Force on Epidemiology and Prevention; National Heart, Lung, and Blood Institute; American Heart Association; World Heart Federation; International Atherosclerosis Society; and International Association for the Study of Obesity. Circulation 2009, 120, 1640-1645. [CrossRef]

2. The IDF Consensus Worldwide Definition of the Metabolic Syndrome. Available online: https://www.idf.org/e-library/ consensus-statements / 60-idfconsensus-worldwide-definitionof-the-metabolic-syndrome.html (accessed on 4 February 2021).

3. Mottillo, S.; Filion, K.B.; Genest, J.; Joseph, L.; Pilote, L.; Poirier, P.; Rinfret, S.; Schiffrin, E.L.; Eisenberg, M.J. The metabolic syndrome and cardiovascular risk a systematic review and meta-analysis. J. Am. Coll. Cardiol. 2010, 56, 1113-1132. [CrossRef] [PubMed]

4. van Meijl, L.E.; Vrolix, R.; Mensink, R.P. Dairy product consumption and the metabolic syndrome. Nutr. Res. Rev. 2008, 21, 148-157. [CrossRef] [PubMed]

5. Unger, A.L.; Torres-Gonzalez, M.; Kraft, J. Dairy Fat Consumption and the Risk of Metabolic Syndrome: An Examination of the Saturated Fatty Acids in Dairy. Nutrients 2019, 11, 2200. [CrossRef]

6. Chen, G.C.; Szeto, I.M.; Chen, L.H.; Han, S.F.; Li, Y.J.; van Hekezen, R.; Qin, L.Q. Dairy products consumption and metabolic syndrome in adults: Systematic review and meta-analysis of observational studies. Sci. Rep. 2015, 5, 14606. [CrossRef] [PubMed]

7. Kim, Y.; Je, Y. Dairy consumption and risk of metabolic syndrome: A meta-analysis. Diabet. Med. 2016, 33, 428-440. [CrossRef]

8. Lee, M.; Lee, H.; Kim, J. Dairy food consumption is associated with a lower risk of the metabolic syndrome and its components: A systematic review and meta-analysis. Br. J. Nutr. 2018, 120, 373-384. [CrossRef] [PubMed]

9. Mena-Sanchez, G.; Becerra-Tomas, N.; Babio, N.; Salas-Salvado, J. Dairy Product Consumption in the Prevention of Metabolic Syndrome: A Systematic Review and Meta-Analysis of Prospective Cohort Studies. Adv. Nutr. 2019, 10, S144-S153. [CrossRef]

10. Korea Centers for Disease Control and Prevention. User Guide for the Sixth Korea National Health and Nutrition Examination Survey (KNHANES VI); Korea Centers for Disease Control and Prevention: Cheongwon, Korea, 2019.

11. Korea Centers for Disease Control and Prevention. User Guide for the Seventh Korea National Health and Nutrition Examination Survey (KNHANES VII); Korea Centers for Disease Control and Prevention: Cheongwon, Korea, 2020.

12. The Korean Nutrition Society; Ministry of Health and Welfare. Dietary Reference Intakes for Koreans 2015: Application Guidebook; Korean Nutrition Society: Seoul, Korea, 2016.

13. Grundy, S.M. Third Report of the National Cholesterol Education Program (NCEP) Expert Panel on Detection, Evaluation, and Treatment of High Blood Cholesterol in Adults (Adult Treatment Panel III) final report. Circulation 2002, 106, $3143-3421$.

14. Seo, M.H.; Lee, W.Y.; Kim, S.S.; Kang, J.H.; Kang, J.H.; Kim, K.K.; Kim, B.Y.; Kim, Y.H.; Kim, W.J.; Kim, E.M.; et al. 2018 Korean Society for the Study of Obesity Guideline for the Management of Obesity in Korea. J. Obes. Metab. Syndr. 2019, 28, 40-45. [CrossRef]

15. Stroup, D.F.; Berlin, J.A.; Morton, S.C.; Olkin, I.; Williamson, G.D.; Rennie, D.; Moher, D.; Becker, B.J.; Sipe, T.A.; Thacker, S.B.; et al. Meta-analysis of Observational Studies in Epidemiology: A Proposal for Reporting. JAMA 2000, 283, 2008-2012. [CrossRef]

16. DerSimonian, R.; Laird, N. Meta-Analysis in Clinical Trials. Control. Clin. Trials 1986, 7, 77-88. [CrossRef]

17. Lawlor, D.A.; Ebrahim, S.; Timpson, N.; Davey Smith, G. Avoiding milk is associated with a reduced risk of insulin resistance and the metabolic syndrome: Findings from the British Women's Heart and Health Study. Diabet. Med. 2005, 22, 808-811. [CrossRef]

18. Jung, H.J.; Han, S.N.; Song, S.; Paik, H.Y.; Baik, H.W.; Joung, H. Association between adherence to the Korean Food Guidance System and the risk of metabolic abnormalities in Koreans. Nutr. Res. Pract. 2011, 5, 560-568. [CrossRef] 
19. Mosley, M.A.; Andrade, F.C.D.; Aradillas-Garcia, C.; Teran-Garcia, M. Consumption of Dairy and Metabolic Syndrome Risk in a Convenient Sample of Mexican College Applicants. Food Nutr. Sci. 2013, 4, 56-65. [CrossRef]

20. Strand, M.A.; Perry, J.; Wang, P.; Liu, S.; Lynn, H. Risk factors for metabolic syndrome in a cohort study in a north China urban middle-aged population. Asia Pac. J. Public Health 2015, 27, NP255-NP265. [CrossRef]

21. Chang, S.H.; Chang, Y.Y.; Wu, L.Y. Gender differences in lifestyle and risk factors of metabolic syndrome: Do women have better health habits than men? J. Clin. Nurs. 2019, 28, 2225-2234. [CrossRef] [PubMed]

22. Pereira, M.A.; Jacobs, D.R.J.; Van Horn, L.; Slattery, M.L.; Kartashov, A.I.; Ludwig, D.S. Dairy consumption, obesity, and the insulin resistance syndrome in young adults. JAMA 2002, 287, 2081-2089. [CrossRef] [PubMed]

23. Mirmiran, P.; Moslehi, N.; Hosseinpanah, F.; Sarbazi, N.; Azizi, F. Dietary determinants of unhealthy metabolic phenotype in normal weight and overweight/obese adults: Results of a prospective study. Int. J. Food Sci. Nutr. 2020, 71, 891-901. [CrossRef]

24. Cheraghi, Z.; Nedjat, S.; Mirmiran, P.; Moslehi, N.; Mansournia, N.; Etminan, M.; Mansournia, M.A.; McCandless, L.C. Effects of food items and related nutrients on metabolic syndrome using Bayesian multilevel modelling using the Tehran Lipid and Glucose Study (TLGS): A cohort study. BMJ Open 2018, 8, e020642. [CrossRef]

25. Beydoun, M.A.; Gary, T.L.; Caballero, B.H.; Lawrence, R.S.; Cheskin, L.J.; Wang, Y. Ethnic differences in dairy and related nutrient consumption among US adults and their association with obesity, central obesity, and the metabolic syndrome. Am. J. Clin. Nutr. 2008, 87, 1914-1925. [CrossRef]

26. Cochran, W.G. The combination of estimates from different experiments. Biometrics 1954, 10, 101-129. [CrossRef]

27. Higgins, J.P.; Thompson, S.G.; Deeks, J.J.; Altman, D.G. Measuring inconsistency in meta-analyses. BMJ 2003, 327, 557-560. [CrossRef]

28. Greenland, S.; Longnecker, M.P. Methods for trend estimation from summarized dose-response data, with applications to meta-analysis. Am. J. Epidemiol. 1992, 135, 1301-1309. [CrossRef]

29. Berlin, J.A.; Longnecker, M.P.; Greenland, S. Meta-analysis of epidemiologic dose-response data. Epidemiology 1993, 4, 218-228. [CrossRef] [PubMed]

30. Orsini, N.; Bellocco, R.; Greenland, S. Generalized least squares for trend estimation of summarized dose-response data. Stata J. 2006, 6, 40-57. [CrossRef]

31. Bodner-Montville, J.; Ahuja, J.K.C.; Ingwersen, L.A.; Haggerty, E.S.; Enns, C.W.; Perloff, B.P. USDA Food and Nutrient Database for Dietary Studies: Released on the web. J. Food Compos. Anal. 2006, 19, S100-S107. [CrossRef]

32. Begg, C.B.; Mazumdar, M. Operating characteristics of a rank correlation test for publication bias. Biometrics 1994, 50, $1088-1101$. [CrossRef]

33. Egger, M.; Smith, G.D.; Schneider, M.; Minder, C. Bias in metaanalysis detected by a simple, graphical test. BMJ 1997, 315, 629-634. [CrossRef]

34. Damião, R.; Castro, T.G.; Cardoso, M.A.; Gimeno, S.G.A.; Ferreira, S.R.G. Dietary intakes associated with metabolic syndrome in a cohort of Japanese ancestry. Br. J. Nutr. 2006, 96, 532-538. [CrossRef]

35. Lutsey, P.L.; Steffen, L.M.; Stevens, J. Dietary intake and the development of the metabolic syndrome: The Atherosclerosis Risk in Communities study. Circulation 2008, 117, 754-761. [CrossRef]

36. Snijder, M.B.; van Dam, R.M.; Stehouwer, C.D.; Hiddink, G.J.; Heine, R.J.; Dekker, J.M. A prospective study of dairy consumption in relation to changes in metabolic risk factors: The Hoorn Study. Obesity 2008, 16, 706-709. [CrossRef]

37. Duffey, K.J.; Gordon-Larsen, P.; Steffen, L.M.; Jacobs, D.R., Jr.; Popkin, B.M. Drinking caloric beverages increases the risk of adverse cardiometabolic outcomes in the Coronary Artery Risk Development in Young Adults (CARDIA) Study. Am. J. Clin. Nutr. 2010, 92, 954-959. [CrossRef]

38. Fumeron, F.; Lamri, A.; Abi Khalil, C.; Jaziri, R.; Porchay-Baldérelli, I.; Lantieri, O.; Vol, S.; Balkau, B.; Marre, M.; the Data from the Epidemiological Study on the Insulin Resistance Syndrome (DESIR) Study Group. Dairy Consumption and the Incidence of Hyperglycemia and the Metabolic Syndrome. Diabetes Care 2011, 34, 813-817. [CrossRef] [PubMed]

39. Lin, Y.-H.; Chang, H.-T.; Tseng, Y.-H.; Lin, M.-H.; Chen, Y.-C.; Yang, H.-W.; Chen, T.-J.; Hwang, S.-J. Characteristics and Health Behavior of Newly Developed Metabolic Syndrome Among Community-Dwelling Elderly in Taiwan. Int. J. Gerontol. 2013, 7 , 90-96. [CrossRef]

40. Louie, J.C.; Flood, V.M.; Rangan, A.M.; Burlutsky, G.; Gill, T.P.; Gopinath, B.; Mitchell, P. Higher regular fat dairy consumption is associated with lower incidence of metabolic syndrome but not type 2 diabetes. Nutr. Metab. Cardiovasc. Dis. 2013, 23, 816-821. [CrossRef] [PubMed]

41. Babio, N.; Becerra-Tomas, N.; Martinez-Gonzalez, M.A.; Corella, D.; Estruch, R.; Ros, E.; Sayon-Orea, C.; Fito, M.; Serra-Majem, L.; Aros, F.; et al. Consumption of Yogurt, Low-Fat Milk, and Other Low-Fat Dairy Products Is Associated with Lower Risk of Metabolic Syndrome Incidence in an Elderly Mediterranean Population. J. Nutr. 2015, 145, 2308-2316. [CrossRef] [PubMed]

42. Sayon-Orea, C.; Bes-Rastrollo, M.; Marti, A.; Pimenta, A.M.; Martin-Calvo, N.; Martinez-Gonzalez, M.A. Association between yogurt consumption and the risk of metabolic syndrome over 6 years in the SUN study. BMC Public Health 2015, 15, 170. [CrossRef] [PubMed]

43. Kim, D.; Kim, J. Dairy consumption is associated with a lower incidence of the metabolic syndrome in middle-aged and older Korean adults: The Korean Genome and Epidemiology Study (KoGES). Br. J. Nutr. 2017, 117, 148-160. [CrossRef] 
44. Beydoun, M.A.; Fanelli-Kuczmarski, M.T.; Beydoun, H.A.; Dore, G.A.; Canas, J.A.; Evans, M.K.; Zonderman, A.B. Dairy product consumption and its association with metabolic disturbance in a prospective study of urban adults. Br. J. Nutr. 2018, 119, 706-719. [CrossRef]

45. Mennen, L.I.; Lafay, L.; Feskens, E.J.M.; Novak, M.; Lépinay, P.; Balkau, B. Possible protective effect of bread and dairy products on the risk of the metabolic syndrome. Nutr. Res. 2000, 20, 335-347. [CrossRef]

46. Azadbakht, L.; Mirmiran, P.; Esmaillzadeh, A.; Azizi, F. Dairy consumption is inversely associated with the prevalence of the metabolic syndrome in Tehranian adults. Am. J. Clin. Nutr. 2005, 82, 523-530. [CrossRef]

47. Liu, S.; Song, Y.; Ford, E.S.; Manson, J.E.; Buring, J.E.; Ridker, P.M. Dietary calcium, vitamin D, and the prevalence of metabolic syndrome in middle-aged and older U.S. women. Diabetes Care 2005, 28, 2926-2932. [CrossRef]

48. Elwood, P.C.; Pickering, J.E.; Fehily, A.M. Milk and dairy consumption, diabetes and the metabolic syndrome: The Caerphilly prospective study. J. Epidemiol. Community Health 2007, 61, 695-698. [CrossRef] [PubMed]

49. Ruidavets, J.B.; Bongard, V.; Dallongeville, J.; Arveiler, D.; Ducimetiere, P.; Perret, B.; Simon, C.; Amouyel, P.; Ferrieres, J. High consumptions of grain, fish, dairy products and combinations of these are associated with a low prevalence of metabolic syndrome. J. Epidemiol. Community Health 2007, 61, 810-817. [CrossRef]

50. Kwon, H.T.; Lee, C.M.; Park, J.H.; Ko, J.A.; Seong, E.J.; Park, M.S.; Cho, B. Milk intake and its association with metabolic syndrome in Korean: Analysis of the third Korea National Health and Nutrition Examination Survey (KNHANES III). J. Korean Med. Sci. 2010, 25, 1473-1479. [CrossRef]

51. Kim, J. Dairy food consumption is inversely associated with the risk of the metabolic syndrome in Korean adults. J. Hum. Nutr. Diet 2013, 26 (Suppl. S1), 171-179. [CrossRef]

52. Huo Yung Kai, S.; Bongard, V.; Simon, C.; Ruidavets, J.B.; Arveiler, D.; Dallongeville, J.; Wagner, A.; Amouyel, P.; Ferrieres, J. Low-fat and high-fat dairy products are differently related to blood lipids and cardiovascular risk score. Eur. J. Prev. Cardiol. 2014, 21, 1557-1567. [CrossRef] [PubMed]

53. Sadeghi, M.; Khosravi-Boroujeni, H.; Sarrafzadegan, N.; Asgary, S.; Roohafza, H.; Gharipour, M.; Sajjadi, F.; Khalesi, S.; RafieianKopaei, M. Cheese consumption in relation to cardiovascular risk factors among Iranian adults IHHP Study. Nutr. Res. Pract. 2014, 8, 336-341. [CrossRef] [PubMed]

54. Martins, M.L.; Kac, G.; Silva, R.A.; Bettiol, H.; Barbieri, M.A.; Cardoso, V.C.; Silva, A.A. Dairy consumption is associated with a lower prevalence of metabolic syndrome among young adults from Ribeirao Preto, Brazil. Nutrition 2015, 31, 716-721. [CrossRef]

55. Drehmer, M.; Pereira, M.A.; Schmidt, M.I.; Alvim, S.; Lotufo, P.A.; Luft, V.C.; Duncan, B.B. Total and Full-Fat, but Not Low-Fat, Dairy Product Intakes are Inversely Associated with Metabolic Syndrome in Adults. J. Nutr. 2016, 146, 81-89. [CrossRef]

56. Falahi, E.; Roosta, S.; Abedini, M.; Ebrahimzadeh, F. Relationship between yoghurt consumption and components of metabolic syndrome: A cross-sectional study in the west of Iran. Int. Dairy J. 2016, 61, 85-90. [CrossRef]

57. Guo, H.; Gao, X.; Ma, R.; Liu, J.; Ding, Y.; Zhang, M.; Zhang, J.; Mu, L.; He, J.; Yan, Y.; et al. Prevalence of Metabolic Syndrome and its Associated Factors among Multi-ethnic Adults in Rural Areas in Xinjiang, China. Sci. Rep. 2017, 7, 17643. [CrossRef] [PubMed]

58. Kim, O.Y.; Kwak, S.Y.; Kim, B.; Kim, Y.S.; Kim, H.Y.; Shin, M.J. Selected Food Consumption Mediates the Association between Education Level and Metabolic Syndrome in Korean Adults. Ann. Nutr. Metab. 2017, 70, 122-131. [CrossRef] [PubMed]

59. Mahanta, T.G.; Joshi, R.; Mahanta, B.; Gogoi, P. Determinants of metabolic syndrome (MetS) amongst persons living in Dibrugarh District of Assam. Clin. Epidemiol. Glob. Health 2017, 5, 52-61. [CrossRef]

60. Shin, S.; Lee, H.W.; Kim, C.E.; Lim, J.; Lee, J.K.; Kang, D. Association between Milk Consumption and Metabolic Syndrome among Korean Adults: Results from the Health Examinees Study. Nutrients 2017, 9, 1102. [CrossRef] [PubMed]

61. Bhavadharini, B.; Dehghan, M.; Mente, A.; Rangarajan, S.; Sheridan, P.; Mohan, V.; Iqbal, R.; Gupta, R.; Lear, S.; Wentzel-Viljoen, E.; et al. Association of dairy consumption with metabolic syndrome, hypertension and diabetes in 147812 individuals from 21 countries. BMJ Open Diabetes Res. Care 2020, 8, e000826. [CrossRef] [PubMed]

62. Hidayat, K.; Yu, L.G.; Yang, J.R.; Zhang, X.Y.; Zhou, H.; Shi, Y.J.; Liu, B.; Qin, L.Q. The association between milk consumption and the metabolic syndrome: A cross-sectional study of the residents of Suzhou, China and a meta-analysis. Br. J. Nutr. 2020, 123, 1013-1023. [CrossRef]

63. Mohammadifard, N.; Akhavan-Tabib, A.; Sajjadi, F.; Alikhasi, H.; Taheri, M.; Khani, A.; Karimi, S. Dairy products and metabolic syndrome among Iranian adult population: Isfahan Healthy Heart Program. Int. Dairy J. 2020, 105, 104667. [CrossRef]

64. Pasdar, Y.; Moradi, S.; Esfahani, N.H.; Darbandi, M.; Niazi, P. Intake of Animal Source Foods in Relation to Risk of Metabolic Syndrome. Prev. Nutr. Food Sci. 2020, 25, 133-139. [CrossRef] [PubMed]

65. Jin, S.; Je, Y. Dairy Consumption and Risk of Metabolic Syndrome in Korean Adults and Elderly People. Metab. Syndr. Relat. Disord. 2020. [CrossRef] [PubMed]

66. Lee, M.S.; Wahlqvist, M.L.; Peng, C.J. Dairy foods and health in Asians: Taiwanese considerations. Asia Pac. J. Clin. Nutr. 2015, 24 (Suppl. S1), S14-S20. [CrossRef] [PubMed]

67. OECD/FAO. Dairy and dairy products. In OECD-FAO Agricultural Outlook 2019-2028; OECD: Paris, France, 2019. [CrossRef]

68. Park, S.H.; Lee, K.S.; Park, H.Y. Dietary carbohydrate intake is associated with cardiovascular disease risk in Korean: Analysis of the third Korea National Health and Nutrition Examination Survey (KNHANES III). Int. J. Cardiol. 2010, 139, 234-240. [CrossRef]

69. Park, S.J.; Park, J.; Song, H.J.; Lee, C.H.; Lee, H.J. Association between dairy product intake and hypertriglyceridemia in Korean adults. Nutr. Res. Pract. 2020, 14, 152-159. [CrossRef] [PubMed]

70. Taskinen, M.R.; Packard, C.J.; Boren, J. Dietary Fructose and the Metabolic Syndrome. Nutrients 2019, 11, 1987. [CrossRef] 
71. Rice, B.H.; Cifelli, C.J.; Pikosky, M.A.; Miller, G.D. Dairy components and risk factors for cardiometabolic syndrome: Recent evidence and opportunities for future research. Adv. Nutr. 2011, 2, 396-407. [CrossRef]

72. Van Loan, M. The role of dairy foods and dietary calcium in weight management. J. Am. Coll. Nutr. 2009, 28 (Suppl. S1), 120S-129S. [CrossRef] [PubMed]

73. Astrup, A.; Chaput, J.P.; Gilbert, J.A.; Lorenzen, J.K. Dairy beverages and energy balance. Physiol. Behav. 2010, 100, 67-75. [CrossRef]

74. Christensen, R.; Lorenzen, J.K.; Svith, C.R.; Bartels, E.M.; Melanson, E.L.; Saris, W.H.; Tremblay, A.; Astrup, A. Effect of calcium from dairy and dietary supplements on faecal fat excretion: A meta-analysis of randomized controlled trials. Obes. Rev. 2009, 10, 475-486. [CrossRef]

75. Vinarova, L.; Vinarov, Z.; Tcholakova, S.; Denkov, N.D.; Stoyanov, S.; Lips, A. The mechanism of lowering cholesterol absorption by calcium studied by using an in vitro digestion model. Food Funct. 2016, 7, 151-163. [CrossRef]

76. Zemel, M.B. Regulation of adiposity and obesity risk by dietary calcium: Mechanisms and implications. J. Am. Coll. Nutr. 2002, 21, 146S-151S. [CrossRef] [PubMed]

77. Kris-Etherton, P.M.; Grieger, J.A.; Hilpert, K.F.; West, S.G. Milk products, dietary patterns and blood pressure management. J. Am. Coll. Nutr. 2009, 28 (Suppl. S1), 103S-119S. [CrossRef]

78. Zemel, M.B. Calcium modulation of hypertension and obesity: Mechanisms and implications. J. Am. Coll. Nutr. 2001, 20, 428S-435S. [CrossRef] [PubMed]

79. Young, D.B.; Lin, H.; McCabe, R.D. Potassium's cardiovascular protective mechanisms. Am. J. Physiol. 1995, 268, R825-R837. [CrossRef] [PubMed]

80. Huth, P.J.; DiRienzo, D.B.; Miller, G.D. Major Scientific Advances with Dairy Foods in Nutrition and Health. J. Dairy Sci. 2006, 89, 1207-1221. [CrossRef]

81. Fluegel, S.M.; Shultz, T.D.; Powers, J.R.; Clark, S.; Barbosa-Leiker, C.; Wright, B.R.; Freson, T.S.; Fluegel, H.A.; Minch, J.D.; Schwarzkopf, L.K.; et al. Whey beverages decrease blood pressure in prehypertensive and hypertensive young men and women. Int. Dairy J. 2010, 20, 753-760. [CrossRef]

82. Boon, N.; Hul, G.B.; Stegen, J.H.; Sluijsmans, W.E.; Valle, C.; Langin, D.; Viguerie, N.; Saris, W.H. An intervention study of the effects of calcium intake on faecal fat excretion, energy metabolism and adipose tissue mRNA expression of lipid-metabolism related proteins. Int. J. Obes. 2007, 31, 1704-1712. [CrossRef]

83. Lu, W.; Chen, H.; Niu, Y.; Wu, H.; Xia, D.; Wu, Y. Dairy products intake and cancer mortality risk: A meta-analysis of 11 population-based cohort studies. Nutr. J. 2016, 15, 91. [CrossRef]

84. Henagan, T.M.; Stefanska, B.; Fang, Z.; Navard, A.M.; Ye, J.; Lenard, N.R.; Devarshi, P.P. Sodium butyrate epigenetically modulates high-fat diet-induced skeletal muscle mitochondrial adaptation, obesity and insulin resistance through nucleosome positioning. Br. J. Pharmacol. 2015, 172, 2782-2798. [CrossRef]

85. Malapaka, R.R.V.; Khoo, S.; Zhang, J.; Choi, J.H.; Zhou, X.E.; Xu, Y.; Gong, Y.; Li, J.; Yong, E.L.; Chalmers, M.J.; et al. Identification and mechanism of 10-carbon fatty acid as modulating ligand of peroxisome proliferator-activated receptors. J. Biol. Chem. 2012, 287, 183-195. [CrossRef]

86. Sengupta, A.; Ghosh, M. Comparison of native and capric acid-enriched mustard oil effects on oxidative stress and antioxidant protection in rats. Br. J. Nutr. 2012, 107, 845-849. [CrossRef] [PubMed]

87. Hu, F.B.; Stampfer, M.J.; Manson, J.E.; Ascherio, A.; Colditz, G.A.; Speizer, F.E.; Hennekens, C.H.; Willett, W.C. Dietary saturated fats and their food sources in relation to the risk of coronary heart disease in women. Am. J. Clin. Nutr. 1999, 70, 1001-1008. [CrossRef] [PubMed]

88. Jacques, P.F.; Wang, H. Yogurt and weight management. Am. J. Clin. Nutr. 2014, 99, 1229S-1234S. [CrossRef] [PubMed]

89. Ruan, Y.; Sun, J.; He, J.; Chen, F.; Chen, R.; Chen, H. Effect of Probiotics on Glycemic Control: A Systematic Review and Meta-Analysis of Randomized, Controlled Trials. PLoS ONE 2015, 10, e0132121. [CrossRef]

90. Nilsen, R.; Hostmark, A.T.; Haug, A.; Skeie, S. Effect of a high intake of cheese on cholesterol and metabolic syndrome: Results of a randomized trial. Food Nutr. Res. 2015, 59, 27651. [CrossRef]

91. Sun, K.; Ren, M.; Liu, D.; Wang, C.; Yang, C.; Yan, L. Alcohol consumption and risk of metabolic syndrome: A meta-analysis of prospective studies. Clin. Nutr. 2014, 33, 596-602. [CrossRef] [PubMed] 